Editorial

\title{
Acknowledgement to Reviewers of Nanomaterials in 2019
}

\author{
Nanomaterials Editorial Office \\ MDPI, St. Alban-Anlage 66, 4052 Basel, Switzerland \\ Published: 19 January 2020
}

The editorial team greatly appreciates the reviewers who have dedicated their considerable time and expertise to the journal's rigorous editorial process over the past 12 months, regardless of whether the papers are finally published or not. In 2019, a total of 1807 papers were published in the journal, with a median time to first decision of 14 days and a median time from submission to publication of 33 days. The editors would like to express their sincere gratitude to the following reviewers for their generous contribution in 2019:

$\begin{array}{ll}\text { Abatzoglou, Nicolas } & \text { Águeda, Vicente I. } \\ \text { Abbrent, Sabina } & \text { Agustin, Dominique } \\ \text { Abdala, Ahmed } & \text { Ahadian, Samad } \\ \text { Abdala, Paula Macarena } & \text { Ahmad, Zeeshan } \\ \text { Abdal-hay, Abdalla } & \text { Ahmadivand, Arash } \\ \text { Abdelkader, Amor M. } & \text { Ahmadivand, Arash } \\ \text { Abdel-Mohsen, A.M. } & \text { Ahmed, Marya } \\ \text { Abdulhalim, Ibrahim } & \text { Ahmed, Mohammad Boshir } \\ \text { Abramovich, Haim } & \text { Ahn, Byung Tae } \\ \text { Abramovich, Haim } & \text { Ahn, Sanghyun } \\ \text { Abramson, Sébastien } & \text { Àhniyaz, Anwar } \\ \text { Abu-Reziq, Raed } & \text { Akagi, Satoshi } \\ \text { Achilias, Dimitris S. } & \text { Akazawa, Housei } \\ \text { Achim, Cristina } & \text { Akbari Garakani, Mohammad } \\ \text { Acker, Jörg } & \text { Åkermark, Björn } \\ \text { Acres, Robert G. } & \text { Akhlaghi, Shahin } \\ \text { Adachi, Sadao } & \text { Akhtari, Massoud } \\ \text { Adam, Vojtech } & \text { Akita, Sadanori } \\ \text { Adamson, Douglas H. } & \text { Akitsu, Takashiro } \\ \text { Adlhart, Christian } & \text { Akitsu, Takashiro } \\ \text { Adonin, Sergey A. } & \text { Ala, Guido } \\ \text { Aflori, Magdalena } & \text { Alarco, Jose } \\ \text { Afzal, Adeel } & \text { Alavi, Saman } \\ \text { Agarwal, Gunjan } & \text { Al-Bataineh, Sameer } \\ \text { Agarwala, Shweta } & \text { Alberti, Marcos G. } \\ \text { Agnoli, Stefano } & \text { Alcoutlabi, Mataz } \\ \text { Agrawal, Prashansa } & \text { Aldakov, Dmitry } \\ \text { Agrawal, Richa } & \text { Alegre, Cinthia } \\ & \end{array}$


Alegria, Elisabete C.B.A.

Alemán, Carlos

Alemany, Pere

Alessandri, Ivano

Alexiev, Ulrike

Alexiou, Christoph

Algharagholy, Laith

Alhabeb, Mohamed

Alhwaige, Almahdi

Ali Ansari, Sajid

Ali, Monsur

Alikin, Denis

Almeida, Jose

Almoazen, Hassen

Al-Shannaq, Refat

Altmann, Friedrich

Altomare, Marco

Álvarez Centeno, Teresa

Alvarez, José

Álvarez, Mar

Alvarez, Noe T.

Álvaro, Caballero

Alzina Sureda, Francesc

Amarie, Dragos

Amarpuri, Gaurav

Amat, Esteve

Ambrosetti, Alberto

Ameloot, Marcel

Amendola, Vincenzo

Anasori, Babak

Anastasescu, Mihai

Andersen, Christian P.

Andersson, Mats

Ando, Yoshito

Andrade, Luísa

Andreoli, Cristina

Andreou, Chrysafis

Andreou, Chrysafis

Andreozzi, Assunta

Andres, Juan

Andrews, David

Andritsch, Thomas

Andrulevicius, Mindaugas
Ang, Yee Sin

Angelici, Robert J.

Angelova, Angelina

Anicǎi, Liana

Anifandis, George

Aniskevich, Andrey

Anisoara Peptu, Catalina

Annanouch, Fatima Ezahra

Anni, Marco

Annick, Rubbens

Antoine, Rodolphe

Antoniac, Iulian

Antoniadou, Maria

Antonino, Famulari

Antonioli, Diego

Antonopoulou, Io

Antonović, Alan

Aoni, Rifat Ahmmed

Apetrei, Constantin

Apetrei, Constantin

Apollonio, Francesca

Apostol, Marian

Apostoluk, Aleksandra

Aquilano, Dino

Aradilla, David

Aradilla, David

Arakawa, Toshiya

Arandiyan, Hamidreza

Aranguren, Mirta I.

Arao, Yoshihiko

Arao, Yoshihiko

Araujo, Daniel

Arbizzani, Catia

Arena, Antonella

Argyle, Morris

Arias-Pardilla, Joaquín

Ariese, Freek

Ariga, Katsuhiko

Armbruster, Udo

Arnaud, Françoise

Arnold, Donna C.

Arrais, Aldo

Arrieta, Marina 
Arrigo, Rossella

Arroyo, Netzahualcóyotl

Artetxe, Beñat

Asadnia, Mohsen

Asano, Ryutaro

Asayama, Shoichiro

Asensio, Maria-Carmen

Ašmontas, Steponas

Assadi, Aymen

Assender, Hazel

Asztemborska, Monika

Ata, Seisuke

Ataollahi, Narges

Atienzar, Pedro

Atsawasuwan, Phimon

Atuchin, Victor V.

Avarvari, Narcis

Aversa, Lucrezia

Avnir, David

Avolio, Roberto

Avram, Marioara

Awan, Shakil Ahmed

Axelevitch, Alexander

Ayukawa, Yasunori

Ayyavoo, Jayalakshmi

Aznar, Elena

Babarao, Ravichandar

Babeva, Tsvetanka

Babu, Anish

Baccarani, Giorgio

Bacsa, Wolfgang

Badawy, Amro El

Badía Laíño, Rosana

Badia, Jose

Badilescu, Simona

Bae, Changdeuck

Baek, Jong-Suep

Baek, Youngbin

Baeza, Alejandro

Baeza, Mireia

Bagdziunas, Gintautas

Bahr, David

Baimova, Julia A.
Bajda, Tomasz

Baji, Zsófia

Bakhtiyarov, Sayavur

Bakolas, Asterios

Bakonyi, Imre

Balandin, Alexander A.

Balandin, Alexander A.

Balasingam, Suresh Kannan

Balasubramanian, Kannan

Baldi, Franco

Bálint, Erika

Ballarin, Barbara

Ballato, John

Ballesta-Claver, Julio

Ballesté, Ruben Mas

Ballesteros, Belén

Balogh-Weiser, Diana

Balsamo, Marco

Balzer, Frank

Banach, Marcin

Banchelli, Martina

Bandala, Erick R.

Bandyopadhyay, Supriyo

Banfi, Francesco

Bao, Yi

Baranowska, Jolanta

Barawi, Mariam

Barbato, Felicia Carla Tiziana

Barbetta, Andrea

Barbillon, Grégory

Barbillon, Grégory

Barbu-Tudoran, Lucian

Bardi, Giuseppe

Barek, Jiri

Barfidokht, Abbas

Barge, Alessandro

Barille, Regis

Barison, Simona

Barker, Philip J

Barnes, Frank S.

Baron, Marco

Baroutaji, Ahmad

Barretta, Raffaele 


\begin{tabular}{|c|c|}
\hline Barron, Andrew R. & Berlier, Gloria \\
\hline Bartley, Jonathan & Bermudez, Maria \\
\hline Bartolomeo, Antonio Di & Bernard, Geffroy \\
\hline Bartsch, Heike & Bernard, HUMBERT \\
\hline Baruth, Andrew & Bernardi, Sara \\
\hline Baskoutas, Sotirios & Bernasconi, Leonardo \\
\hline Basoli, Francesco & Bernechea, María \\
\hline Batmunkh, Munkhbayar & Bert, Nikolay \\
\hline Batra, A. K. & Bertocci, Francesco \\
\hline Battiato, Alfio & Bertoglio, Federico \\
\hline Batuk, Maria & Bertolotti, Federica \\
\hline Bäumler, Hans & Bertolotti, Mario \\
\hline Bayer, Ilker & Berton, Paula \\
\hline Baykara, Mehmet & Bertoncello, Paolo \\
\hline Bayraktar, Emin & BERUETE, MIGUEL \\
\hline Bazylinska, Urzsula & Bessergenev, Valentin G. \\
\hline Beake, Ben & Betancourt, Tania \\
\hline Bear, Joseph C. & Bettini, Simona \\
\hline Bec, Krzysztof B. & Bettotti, Paolo \\
\hline Becerra, Marley & Beyerlein, Kenneth \\
\hline Bechelany, Mikhael & Bhandari, Khagendra \\
\hline Becucci, Maurizio & Bhattacharjya, Dhrubajyoti \\
\hline Beg, Marijan & Bhattacharya, Sriparna \\
\hline Behar, Joachim & Bianchi, Massimiliano \\
\hline Beker, Levent & Bianco, Vincenzo \\
\hline Bekyarova, Elena & Bielas, Rafał \\
\hline BelBruno, Joseph & Bielecki, Stanisław \\
\hline Beletskaya, Irina Petrovna & Biernat, Jan \\
\hline Belkhou, Rachid & Bigall, Nadja \\
\hline Bella, Federico & Bigiani, Lorenzo \\
\hline Bellardita, Marianna & Bikiaris, Dimitrios \\
\hline Bellos, Evangelos & Bilewicz, Aleksander \\
\hline Bellucci, Stefano & Bini, Fabiano \\
\hline Belsley, Michael Scott & Bini, Marcella \\
\hline Beltsios, K. & Birch, Brian \\
\hline Bender, Florian & Birke, Ronald L. \\
\hline Benedetti, Elisabetta & Bishop, Greg \\
\hline Benes, Hynek & Bison, Georg \\
\hline Benito, Juan M. & Biswas, Kaushik \\
\hline Benoit, Denizot & Bittkau, Karsten \\
\hline Benseman, Timothy M. & Bjork, Emma \\
\hline Benson, Heather & Bjorklund, Robert B. \\
\hline Berenjian, Aydin & Björkman, Torbjörn \\
\hline
\end{tabular}




\begin{tabular}{|c|c|}
\hline Blanco, Angeles & Bossmann, Stefan \\
\hline Blanco, Miren & Boudaden, Jamila \\
\hline Blasi, Paolo & Bouquillon, Sandrine \\
\hline Blau, Werner & Bouranis, Dimitris L. \\
\hline Blayo, Anne & Bourgeois, Olivier \\
\hline Blein, Thomas & Bourillot, Eric \\
\hline Błoch, Katarzyna & Bourlinos, Athanasios B. \\
\hline Block, Stephan & Bouropoulos, Nikolaos \\
\hline Bloh, Jonathan Z. & Bousser, Étienne \\
\hline Bloise, Andrea & Bouyer, Frederic \\
\hline Bludov, Yuliy & Bouziotis, Penelope \\
\hline Bobacka, Johan & Bowen, James \\
\hline Bochenkov, Vladimir E. & Boyd, Anthony \\
\hline Bochkarev, M. N. & Bozo, Eva \\
\hline Boczkaj, Grzegorz & Braga, Susana Santos \\
\hline Boddapati, Loukya & Braic, Mariana \\
\hline Bodzenta, Jerzy & Branicio, Paulo \\
\hline Boeffel, Christine & Branquinho, Rita \\
\hline Boffa, Vittorio & Breslin, Carmel B. \\
\hline Boffito, Monica & Brestic, Marian \\
\hline Boisseau, Sebastien & Breunig, Hans Georg \\
\hline Boix, Pablo P. & Brewer, Samuel M. \\
\hline Bokias, Georgios & Briesen, Heiko \\
\hline Boldrini, Stefano & Briois, Pascal \\
\hline Boles, Corey & Brogioli, Doriano \\
\hline Bolivar, Juan M. & Brookes, Nicholas \\
\hline Boncel, Slawomir & Brosseau, Christian \\
\hline Bonduelle, Colin & Brougham, Dermot \\
\hline Bonferoni, Maria Cristina & Brousse, Thierry \\
\hline Bonifácio, Vasco & Brouzgou, Angeliki \\
\hline Bonnet, Célia S. & Brown, David (France) \\
\hline Bonnet, Pierre-Antoine & Brown, David (UK) \\
\hline Bonyár, Attila & Brown, Katherine \\
\hline Borensztein, Yves & Broza, Yoav \\
\hline Borfecchia, Elisa & Brun, Nicolas \\
\hline Borges, João Paulo & Bruno, Annalisa \\
\hline Borges, Joel & Bruno, Mattos \\
\hline Borin, Dmitry & Brutti, Sergio \\
\hline Borowiec, Joanna & Bryjak, Marek \\
\hline Borriello, Anna & Bubach, Bailey \\
\hline Bortolotto, Tissiana & Bubnov, Alexey \\
\hline Boscá, Francisco & Buckner, Steven W. \\
\hline Boshir Ahmed, Mohammad & Bueken, Bart \\
\hline
\end{tabular}




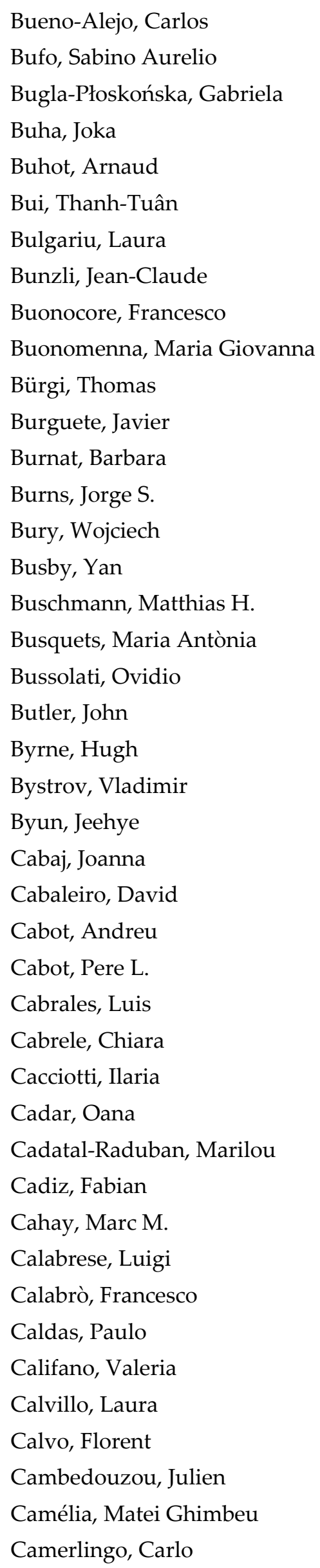

Cameron, David

Cameron, Joseph

Cametti, Cesare

Camilli, Luca

Caminati, Gabriella

Cammas-Marion, Sandrine

Campbell, Kris

Campos-Martínez, J.

Candiani, Gabriele

Caneschi, Andrea

Canet Ferrer, Josep

Canet-Ferrer, Josep

Canetta, Elisabetta

Canfield, Brian

Cangialosi, Daniele

Canle, Moisés

Cannas, Marco

Cannio, Maria

Cantalini, Carlo

Cantele, Giovanni

Cao, Xudong

Capasso, Andrea

Capelli, Sofia

Capeness, Michael

Capiglia, Claudio

Caporali, Stefano

Capsoni, Doretta

Capuano, Rosamaria

Caputo, Gianvito

Caputo, Gregory

Carabineiro, Sónia

Carata, Elisabetta

Carbone, Claudia

Cardia, Maria Cristina

Caridad, Jose

Carli, Stefano

Carlomagno, Ilaria

Carmo, Helena

Carmona, Francisco

Carosio, Federico

Carotenuto, Gianfranco

Carrasco-Marin, Francisco

Cartagena-Rivera, Alexander 
Caruntu, Gabriel

Casado-Coterillo, Clara

Casanove, Marie-Jose

Casassa, Silvia

Caseri, Walter

Casey, Sean

Cassani, Maria Cristina

Cassidy, John F.

Castaldi, Giuseppe

Castaldo, Anna

Castanheira, Elisabete M.S.

Castellanos-Gomez, Andres

Castelli, Ivano E.

Castellino, Micaela

Castillo-Michel, Hiram

Castro, Edison

Casula, Michele

Cataldi, Pietro

Catauro, Michelina

Cauda, Valentina

Cavallaro, Giuseppe

Cavallini, Massimiliano

Cavicchi, Kevin

Cavigli, Lucia

Cazorla-Amorós, Diego

Cazzanelli, Massimo

Cecilia, Juan Antonio

Celano, Umberto

Celebrano, Michele

Celzard, Alain

Centeno, Miguel Angel

Ceresoli, Davide

Cerrato, Giuseppina Pinuccia

Cesano, Federico

Cesário, Rute

Ceseracciu, Luca

Chagnes, Alexandre

Chai, Kyu Yun

Chakraborty, Saumen

Champagne, Benoît

Chandra, Dhyan

Chandrasekaran, Murugesan

Chang, Chia-Chen
Chang, Dong Wook

Chang, Seung-Cheol

Chang, Sheng-Po

Chantana, Jakapan

Charles, Andrew D. M.

Charmas, Barbara

Chassaing, Stefan

Chaudhari, Nitin

Chaudhary, Anna Lisa

Chaudhry, Qasim

Chauhan, Garima

Chava, Rama Krishna

Chavez-Angel, Emigdio

Chen, Binling

Chen, Chia-Yun

Chen, Hone-Zern

Chen, Hui-Yu

Chen, I-Wen Peter

Chen, Jem-Kun

Chen, Jian-Lian

Chen, Jihua

Chen, Jinju

Chen, Kuan-Ren

Chen, Lung-Chien

Chen, Pang-Shiu

Chen, Szu-yuan

Chen, Tao-Hsing

Chen, Wei-Yu

Chen, Yijia

Chen, Yu-Chih

Cheng, Fong-Yu

Cheng, Hsu-Hui

Cheng, Huanyu

Cheng, Ya

Cheraghian, Goshtasp

Cherkaoui, Karim

Chiang, Anthony S.T.

Chiang, Long $Y$

Chiavaioli, Francesco

Chiolerio, Alessandro

Chiono, Valeria

Chiu, Fang-Chyou

Chiu, Te-Wei 
Chizari, Kambiz

Chizhik, Alexey I.

Chmielarz, Paweł

Chmielewski, Andrzej

Cho, Chang-Hee

Cho, Dae-Hyun

Cho, Hanna

Cho, In Sun

Cho, Jung Sang

Cho, Kyu Taek

Cho, Man Ho

Cho, So-Hye

Cho, Sunghun

Choi, Chul-Jin

Choi, Dukhyun

Choi, Hyo-Jick

Choi, Hyoung

Choi, Jae-Hong

Choi, Jane Ru

Choi, Jeong-Woo

Choi, Si-Young

Choi, Yoon-Seuk

Chong, Parskson Lee Gau

Chorazy, Szymon

Chou, Jung Chuan

Chow, James

Chowdhury, Ezharul Hoque

Chrissopoulou, Kiriaki

Christensen, Jørn B

Chronakis, Ioannis S.

Chroneos, Alexander

Chrysikopoulos, Constantinos

Chrzanowski, Wojciech

$\mathrm{Chu}$, Ching-Liang

Chul Wee, Lee

Chun, Sechul

Chung, Chan-Moon

Chung, Dong Young

Chung, Ren-Jei

Chung, Sheng-Heng

Ciampi, Simone

Cicala, Gianluca

Cicciù, Marco
Cinteza, Otilia Ludmila

Ciocan, Corina

Cirillo, Giuseppe

Cizek, Jan

Clarke, Ronald James

Clemens, Oliver

Cobianu, Cornel

Coduri, Mauro

Coelho, Luis

Coelhoso, Isabel

Colace, Lorenzo

Colangelo, Gianpiero

Collins, Scott

Coman, Cristina

Comfort, K K

Compañ Moreno, Vicente

Comparelli, Roberto

Concepción, Patricia

Concetta Valeria Lucia, Giosafatto

Concilio, Simona

Connolly, James P.

Constantin, Lucian

Conte, Claudia

Cordani, Marco

Cordoyiannis, George

Corezzi, Silvia

Cornelius, Thomas Walter

Correa, Cinthia Antunes

Corrigan, Damion K

Cortés, Araceli González

Cortese, Barbara

Cosco, Donato

Cosma, Pinalysa

Costa, Carlos Miguel

Costa, Pedro

Costantino, Ferdinando

Costantino, Luca

Costello, Ben De Lacy

Coudert, Francois-Xavier

Cousin, Renaud

Coutinho, Paula

Coutinho, Paulo José Gomes

Coy, Emerson 
Cozzoli, P. Davide

Cozzolino, Anthony F.

Cragg, Peter

Credico, Barbara Di

Creton, Stuart

Crisp, Ryan W.

Cristallini, Caterina

Cristea, Cecilia

Cristea, Mihaela Dana

Cristian, Petcu

Critchley, Kevin

Croce, Anna Cleta

Croissant, Jonas G.

Crossley, Alison

Crucho, Carina Isabel Correia

Crucianelli, Marcello

Cruz, Sandra

Cruz-Yusta, Manuel

Csík, Attila

Csoka, Levente

Csontos, Miklós

Ctibor, Pavel

Cuberes, Teresa

Cucchiarini, Magali

Culshaw, Brian

Czeppe, Tomasz

Czerwinski, Andrzej

Czigany, Zsolt

Czyż, Jarosław

D'Anna, Francesca

Da Costa Ribeiro, Ana Paula

Da Costa, Ana Rosa

Da Silva, Jaime Pedro Oliveira

Dabrowiak, James

D'accolti, Lucia

Daeneke, Torben

Dagdeviren, Canan

Dahal, Rajendra P

Dal Col, Jessica

D'Alfonso, Laura

Dalmoro, Annalisa

D'Amora, Ugo

Da'na, Enshirah
Daniel-da-Silva, Ana

Daniele, Michael

Danko, Martin

DAnte, Silvia

Danti, Serena

Darling, Seth B.

Darmanin, Connie

Darvell, Brian

Das, Narottam

Das, Sanjib

Dash, Biraja

Daubon, Thomas

David, Iulia Gabriela

Davim, J. Paulo

Davis, Frank

Davis, Fred

Davis, Graham

Dawes, Judith

Dawn, Arnab

Dawson, James A.

Dawson, Jonathan

De Aguiar, José Barroso

De Andrés, Alicia

De Aza, Piedad N.

De Bonis, Angela

De Chaisemartin, Luc

De Falco, Gianluigi

De Gisi, Sabino

De La Escosura-Muñiz, Alfredo

De La Fuente, Germán

De La Rica, Roberto

De Lacalle, Luis N. López

De Luca, Luigi

De Luca, Pierantonio

De Marcellis, Andrea

De Nijs, Bart

De Oliveira, Mario Anderson

De Sio, Luciano

De Sousa, Célia Tavares

De Stefano, Luca

De Vietro, Nicoletta

De Zotti, Marta

Deckert, Alice 
Decroly, André

Deimede, Valadoula

Dejam, Morteza

Del Gaudio, Costantino

Delfino, Ines

Dell'Aglio, Marcella

Del-Rio Celestino, Mercedes

Delville, Marie-Hélène

Demel, Jan

Denat, Franck

Deng, Hai-Qiang

Depciuch, Joanna

D'Errico, Gerardino

Derrien, Thibault

Desmaris, Vincent

Deuss, $P$

Devadas, Mary Sajini

Devasurendra, Amila

DEVEL, Michel

Dhakshinamoorthy, Amarajothi

Dhand, Vivek

Dhanjai, Dhanjai

Dharavath, Srinivas

Dhaygude, H. D

Di Bartolomeo, Antonio

Di Bella, Santo

Di Corato, Riccardo

Di Girolamo, Rocco

Di Marco, Alessandro

Di Martino, Piera

Di Noto, Vito

Diamanti, Maria Vittoria

Diaz Fernandez, Yuri

Díaz, Esperanza

Diba, Mani

Dichiara, Anthony

Dickert, Franz L.

Dickey, Michael

Díez, Noel

Díez-Pascual, Ana María

Dikin, Dmitriy A.

Dillert, Ralf

Dimakis, Nicholas
Dimitratos, Nikolaos

Dimitrievska, Mirjana

Dimos, Konstantinos

Dina, Nicoleta Elena

Dinca, Valentina

Dinh, Toan

Dinh, Van Tuan

Dini, Danilo

Dini, Luciana

Dinischiotu, Anca

Dinu, Maria Valentina

Diring, Stephane

Distaso, Monica

Dixon, David A.

DJANASHVILI, Kristina

Do Rego, Ana Maria P. L. R. Botelho

Dobrovolskaia, Marina

Dobrzynski, Piotr

Docoslis, Aris

Dodds, Chris

Dohnal, Fadi

Dolocan, Andrei

Domb, Avi

Dombek, Grzegorz

Domenici, Valentina

Domine, Marcelo E.

Dominguez, Carmen M.

Dong, Yajie

Donnet, Christophe

Dorosz, Dominik

Dorozhkin, Sergey V.

Dorp, Dennis Van

Dos Santos-García, Antonio Juan

Doustkhah, Esmail

Dressel, Martin

Drevet, Richard

Drewniak, Sabina

Drikakis, Dimitris

Drisko, Glenna

Drlica, Karl

Duboz, Jean-Yves

Duguet, Etienne

Dulski, Mateusz 
Dumee, Ludovic

Dumitran, Laurenţiu Marius

Dumitrica, Traian

Dumont, Pierre

Dumur, Frédéric

Durach, Maxim

Durante, Christian

Durinck, Julien

Durndell, Lee

Dušková-Smrčková, Miroslava

Duta, Liviu

Dutot, Mélody

Dutta, Kingshuk

Duval, Raphaël E.

Düzgünes, Nejat

Dvoyashkin, Muslim

Dyatkin, Boris

Dyck, Ondrej E.

Dyzia, Maciej

Dzakpasu, Mawuli

Dzantiev, B. B.

Dzida, Marzena

Dziedzic, Arkadiusz

Dziubek, Kamil

Eason, David

Ebara, Mitsuhiro

Economopoulos, Solon P.

Economou, Demetre

Edgar, James $\mathrm{H}$.

Edlund, Ulrica

Efimov, Alexander

Efstathopoulos, Efstathios P.

Eftekhari, Ali

Eftimie Totu, Eugenia

Egawa, Yuya

Egberts, Philip

Egli, Ramon

Ehrmann, Andrea

Einarsson, Erik

Eklund, Per

Ekuni, Daisuke

Eleni, Makarona

El-Gendy, Ahmed A.
Eligiusz, Postek

Ellahi, Rehmat

El-Moghazy, Ahmed

El-Safty, Sherif

Emoto, Makoto

Endoh, Tamaki

Enesca, Ioan Alexandru

Engelhart, Aaron

Engelke, Hanna

Engqvist, Håkan

Ennas, Guido

Ennen, Inga

Enrico, Gherlone Felice

Enríquez Pérez, Esther

Enyashin, Andrey

Epifani, Mauro

Erbe, Artur

Ercole, Francesca

Erdem, Emre

Eriksen, René Lynge

Ermini, Maria Laura

Ermolina, Irina

Erwan, Paineau

Escorihuela, Jorge

Esen, Cemal

Eslami, Hossein

Esparza, Pedro

Espiña, Begoña

Esposito Corcione, Carola

Esposito, Elisabetta

Esposito, Gennaro

Esposito, Serena

Espro, Claudia

Estellé, Patrice

Estelrich, Joan

Esteves Da Silva, Joaquim C.G.

Estrany, Francesc

Etacheri, Vinodkumar

Etienne, Mathieu

Ettayapuram Ramaprasad, Azhagiya Singam

Eubank, Jarrod F.

Euchner, Holger

Eugenio, María E. 
Evangelisti, Claudio

Evans, Christopher

Evlyukhin, Andrey B.

Evtugyn, Gennady

Fabbri, Filippo

Fabiano, Eduardo

Fabio, Marmottini

Fabisiak, Kazimierz

Fabre, Bruno

Fàbrega, Lourdes

Fagadar-Cosma, Eugenia

Fagan, Jeffrey

Faggio, Giuliana

Faia, Pedro

Fajín, José L. C.

Fakharuddin, Azhar

Fakhrullin, Rawil F.

Falco, Alberto

Fales, Andrew

Faley, Michael

Falko, Vladimir

Fancher, Chris M.

Fanciulli, Carlo

Fang, Cheng

Fang, Ronnie H.

Farajpour, Ali

Fare, Silvia

Farè, Silvia

Farka, Zdeněk

Farris, Stefano

Farrugia, Brooke

Fasano, Matteo

Fateixa, Sara Isabel Augusto

Faulkner, R. G.

Favre-Réguillon, Alain

Fazzolari, Fiorenzo

Feczkó, Tivadar

Fedorov, Georgy

Feis, Alessandro

Felgueiras, Helena

Felicetti, Federica

Félix, Luis

Feliz, Marta
Felner, Israel

Fenyvesi, Eva

Fernandes, Maria Helena

Fernandes, Mariana Sofia Peixoto

Fernández Ramos, María Dolores

Fernández, Adolfo

Fernández, José A.

Fernández-García, Marta

Fernández-Romero, Juan Manuel

Ferrara, Chiara

Ferrari, Luc

Ferrari, Maurizio

Ferrari, Maurizio

Ferrari, Michele

Ferraris, Sara

Ferraro, Pietro

Ferreira, Helena

Ferreira, Jose M.F.

Ferreira, Quirina

Ferreiro, Anxo Fernández

Ferrer, Belen

Ferrero, Guillermo Á.

Ferretti, Anna Maria

Fiandra, Luisa

Fic, Krzysztof

Ficek, Mateusz

Fierro, Vanessa

Filgueira, Carly

Filip, Jaroslav

Filipovic, Nenad

Finney, Eric E.

Fioravanti, Ambra

Fiorenza, Patrick

Fioretti, Bernard

Fiori, Stefano

Fiorillo, Antonino

Fiorillo, Fausto

Fischer, Holger

Flandin, Lionel

Flater, Erin E.

Flieger, Jolanta

Florczyk, Stephen

Florea, Ileana 
Floris, Francesco

Flox, Cristina

Focsan, Monica

Foelske, Annette

Fologea, Daniel

Fomin, Vladimir M.

Fonseca, Ana

Font, Francesc

Fontana, Antonella

Fontana, Flavia

Fontanesi, Claudio

Fonte, Pedro

Formela, Krzysztof

Fornasiero, Paolo

Fornell, Jordina

Forner, Leopoldo

Fortenberry, Ryan

Fortuna, Ana

Fortunati, Ilaria

Fortunato, Giuseppino

Foster, Christopher W.

Fracasso, Giulio

Fraga-García, Paula

Fragoso, Alex

Fraix, Aurore

Francés-Soriano, Laura

Francia, Carlotta

Franco, Alessandro

Frank, Irmgard

Frankcombe, Terry

Franssila, Sami

Fratoddi, Ilaria

Frattini, Domenico

Frederick, Brian G.

Freedman, Kevin

Freeman, Eric

Frey, Margaret

Friák, Martin

Frielinghaus, Henrich

Frisenda, Riccardo

Fröhlich, Eleonore

Frontistis, Zacharias

Fuierer, Paul
Fujii, Takenori

FUJIWARA, Akihiko

Fukuda, Takeshi

Fulvio, Pasquale Fernando

Furumi, Seiichi

Furusawa, Hiroyuki

Furuta, Hiroshi

Fuso, Francesco

Gabilondo, Nagore

Gaczynska, Maria

Gadde, Suresh

Gadisa, Abay Dinku

Gadomski, Adam

Gaffet, Eric

Gafurov, Marat

Gago, Sandra

Galamboš, Michal

Galetti, Maricla

Galian, Raquel E.

Galinetto, Pietro

Gallei, Markus

Galstyan, Vardan

Gambardella, Alessandro

Gamelas, José

Gammer, Christoph

Gamsjäger, Ernst

Gane, Patrick

Ganesan, Palanivel

Gangishetty, Mahesh

Ganguly, Pritam

Ganikhanov, Feruz

Ganta, Deepak

Gantenbein, Benjamin

Gao, Nan

Gao, Xuefei

Gao, Yongfeng

Garbovskiy, Yuriy

García Rodríguez, Juan

García Ruiz, Francisco Javier

Garcia, Andres

Garcia, Belen

Garcia, Diana Vilela

Garcia, Iñaki 
García, Nuria

García-Lastra, Juan María

García-López, Elisa I.

García-Martinez, Joaquín C.

Garcia-Pomar, Juan Luis

Garcia-Sanchez, Francisco

Garcia-Segura, Sergi

Garcia-Torres, José

Garcia-Verdugo, Ignacio

Gardiner, Philip

Garg, Akhil

Gargiulo, Valentina

Garmire, Elsa

Garro, Núria

Garroni, Sebastiano

Garshev, Alexey A.

Gartia, Manas Ranjan

Gashti, Mazeyar Parvinzadeh

Gaskov, Alexander M.

Gaspar, Maria Manuela

Gatica, José M.

Gatti, Antonietta

Gatto, Emanuela

Gauden, Piotr A.

Gaudry, Émilie

Gautam, Siddharth

Gavioli, Luca

Gavrilov, Anton

Gebel, Thomas

Gebicki, Jacek

Géczy, Attila

Geier, Sebastian M.

Gelbstein, Yaniv

Geletii, Yurii

Gellini, Cristina

Genovese, Damiano

Gentile, Gennaro

Georgantzinos, Stelios K.

George, Chandramohan

George, Janine

George, Thomas F.

Georgieva, Radostina

Georgiou, T. K.
Georgopanos, Prokopios

Gerasimov, Evgeniy Yu.

Gerasimova, Yulia V.

Gerislioglu, Burak

Gervais, François

Geso, Moshi

Ghanotakis, Demetrios F

Gheju, Marius

Ghibaudo, Gérard

Ghica, Daniela

Ghica, Mihaela-Violeta

Ghidelli, Matteo

Ghittorelli, Matteo

Ghodake, Gajanan

Ghorbani-Asl, Mahdi

Giacalone, Francesco

Giacomazza, Daniela

Giamblanco, Nicoletta

Giancane, Gabriele

Giannakas, Aris

Giannazzo, Filippo

Giannetti, Ambra

Giapintzakis, John (Ioannis)

Giaume, Domitille

Gibson, Christopher

Giebeler, Lars

Gierszewska, Magdalena

Gigliotti, Casimiro Luca

Gil, Bernard

Gillan, Edward G.

Giorcelli, Mauro

Giorgio, Ivan

Girard, Steven

Giraud, Marie Noëlle

Girish, Rughoobur

Girolami, Marco

Gîrțu, Mihai A.

Giubileo, Filippo

Giulia, Fioravanti

Giusti, Ilaria

Gizdavic-Nikolaidis, Marija

Glavina, Domagoj

Gliemann, Hartmut 
Głuchowski, Paweł

Glukhova, Olga E.

Glüsen, Andreas

Gmurek, Marta

Gnade, Bruce E.

Gnaneswar Gude, Veera

Gnyba, Marcin

Gogneau, Noelle

Gogos, Alexander

Golding, Jon

Goldoni, Andrea

Gomes, Ana P.

Gomes, Andreia

Gomes, João

Gomez de Salazar, José María

Gómez-Cámer, Juan Luis

Gómez-Graña, Sergio

Gomez-Ruiz, Santiago

Gonçalves, M. Sameiro T.

Gonçalves, Renato

Gonzalez Vila, Alvaro

González, Israel

Gonzalez, Joaquin

Gonzalez, Zoraida

González-Cortés, Araceli

González-Marcos, María P.

Gonzalez-Pedro, Victoria

Gonzalez-Velo, Yago

Goodwin, David G.

Gopalakrishnan, Kothandam

Gopalan, Saianand

Goraus, Jerzy

Goreham, Renee

Gorgieva, Selestina

Gorji, Nima E.

Gornakov, Vladimir

Gorte, Raymond J.

Goryacheva, Irina

Gorzelanny, Christian

Gosselet, Fabien

Goswami, Subhadip

Goto, Yosuke

Gouget, Guillaume
Goula, Maria A.

Gouveia, Ricardo M.

Govan, Joseph

Govind Rao, Vishal Govind

Gowans, Eric

Gracin, Davor

Graczyk-Jarzynka, Agnieszka

Grama, Silvia

Grammatikopoulos, Panagiotis

Grancha, Thais

Granda, Marcos

Grande Tovar, Carlos David

Granitzer, Petra

Granozzi, Gaetano

Grassi, Alfonso

Grau, Etienne

Gravalidis, Christoforos

Gravel, Edmond

Graves, Joseph

Grebel, Haim

Grecco, Giuseppe

Gregoratti, Luca

Gregori, Alberto

Grenha, Ana

Gries, Thomas

Grigoras, Kestutis

Grigsby, Christopher L.

Grijalvo, Santiago

Grishin, Maxim

Grossi, Marco

Grugeon, Sylvie

Grumezescu, Alexandru

Grunwald, Ruediger

Gruszecka, Agnieszka

Gspann, Thurid

Guaraldo, Thais Tasso

Guarnieri, Simone

Guarrotxena Arlunduaga, Miren Nekane

Gucci, Francesco

Guénin, Erwann

Gueorguiev, G.K.

Gueorguiev, Gueorgui

Guerrero-Perez, M. Olga 
Guest, Jeffrey R.

Guha, Suchi

Guidotti, Matteo

Guiot, Caterina

Guisbiers, Gregory

Gulino, Antonino

Gumfekar, Sarang

Guo, Zhan-Hu

Gupta, Chirag

Gupta, Ram K.

Gupta, Sanju

Gurikov, Pavel

Guselnikova, Olga Andreevna

Gusev, Alexander

Gusiatin, Mariusz

Gutekunst, Will R.

Gutiérrez, Yael

Gutleb, Arno

Guzmán, Eduardo

Guzman, Marcelo

György, Enikö

H. Müser, Prof. Dr. Martin

Ha, Minjeong

Haber, Louis

Hackenberg, Stephan

Haddadi, Abbas

Hadipour, Afshin

Hadjiargyrou, Michael

Hadjikakou, Sotiris K

Hagita, Katsumi

Hahn, Konstanze R.

Hähner, Georg

Hak, Sjoerd

Halder, Arnab

Hallett-Tapley, Geniece

Hama, Susumu

Hämäläinen, Jani

Hampp, Norbert A.

Hampton, Jennifer

Hampton, Michael

Han, Dong-Pyo

Han, Dong-Wook

Han, Eric
Hanaor, Dorian

Hanaor, Dorian A. H.

Handoko, Albertus

Hanif, Muhammad

Haniu, Hisao

Hao, Jinsong

Hara, Kenji

Hara, Masahiro

Haraguchi, Naoki

Haralampos N, Miras

Harja, Maria

Härmä, Harri

Harmandaris, Vagelis A.

Harnois, Maxime

Haro, Marta

Harrell, William R.

Harris, Peter

Hartnagel, Hans

Hasanzadeh Kafshgari, Morteza

Hashimoto, Makoto

Hatachi, Yukimasa

Hatada, Ruriko

Hatakeyama, Kazuto

Hatta, Akimitsu

Hattar, Khalid

Hattori, Azusa N.

Hattori, Haroldo T.

Hatziantoniou, Sophia

Hatzikraniotis, Evripidis

Hawash, Zafer

Hawes, Chris S.

Hayakawa, Tohru

Hayashi, K.

Hayashi, Takuya

$\mathrm{He}$, Aina

He, Delong

$\mathrm{He}$, Jie

Hedberg, Jonas

Hefferon, Kathleen Laura

Hegedűs, Csaba

Hegedűs, László

Hejna, Aleksander

Hellweg, Thomas 
Helsch, Gundula

Hémadi, Miryana

Hempel, Martin

Hemraj-Benny, Tirandai

Henderson, Luke C.

Hendrix, Justin W.

Herckes, Pierre

Hermerschmidt, Felix

Hermoso-Orzáez, Manuel Jesús

Herranz, Fernando

Herrasti Gonzales, Pilar

Herrera-Melián, José Alberto

Herrmann, Andreas

Heuken, Michael

Hibino, Takashi

Higashi, Masanobu

Higginbotham Duque, Amanda L.

Hill, Daniel

Hilliou, Loic

Hinchet, Ronan

Hinderliter, Brian

Hintzen, Hubertus

Hippler, Rainer

Hirano, Atsushi

Hirsch, Thomas

Hix, Gary

Hjelme, Dag Roar

Hnát, Jaromír

Ho, Daniel

Ho, Tuananh

Hobbie, Erik K.

Hodoroaba, Vasile-Dan

Hoffman, Jason

Hoffmann-Eifert, Susanne

Hofman-Caris, Roberta

HogenEsch, Harm

HOLADE, Yaovi

Holler, Stephen

Holmes, Mark

Holynska, Malgorzata

Homaeigohar, Shahin

Honda, Takashi

Hong, Suck Won
Hong, Sukjoon

Hoppstädter, Jessica

Horak, Daniel

Horino, Yoshikazu

Hornak, Jaroslav

Horovistiz, Ana

Horszczaruk, Elżbieta

Hoskins, Clare

Hospodková, Alice

Hossain, Shahriar A.

Hou, Harvey

Hou, Shuhn-Shyurng

Housecroft, Catherine

Houska, Jiri

Howes, Philip

Hoyos, Pilar

Hristoforou, Evangelos

Hsiang, Hsing-I

Hsiao, Chung-Der

Hsiao, Vincent K.S.

Hsieh, Chien-Kuo

Hsieh, Chien-Wen

Hsu, Yu-Kuei

Hsueh, Yi-Huang

$\mathrm{Hu}$, Teh-Min

Huang, Chia-Hua

Huang, Chih-Chia

Huang, Chih-Ching

Huang, Kuo-Cheng

Huang, Ruomeng

Huang, Shih-Yung

Huang, Wen Chang

Hubbe, Martin A.

Hueso, Jose L.

Hughes, Robert A.

Huguet, Silvia Soria

Humar, Miha

Humbert, Christophe

HUMELNICU, Doina

Hung, Tai-Feng

Huple, Deepak

Hur, Jaehyun

Húska, Dalibor 


\begin{tabular}{|c|c|}
\hline Hussain, Manwar & Isaacs, Mark \\
\hline Huttel, Yves & Isaad, Jalal \\
\hline Huynh, Thanh-Canh & Isakov, Dmitry \\
\hline Hwang, Chi-Sun & Isalgue, Antonio \\
\hline Hwang, Sungyeon & Iseppi, Ramona \\
\hline Hwang, Taek Yong & Ishihara, Kazuhiko \\
\hline Hytönen, Vesa & Ishii, Ayumi \\
\hline Iacovella, Christopher R. & Ishikawa, Kenji \\
\hline Ianchis, Raluca & Ishikawa, Ryo \\
\hline Iannazzo, Daniela & Ishikawa, Tadahiko \\
\hline Iatrou, Hermis & Ishikawa, Yasuaki \\
\hline Iatsunskyi, Igor R & Ishizaki, Kenji \\
\hline Ibrahim, Toni & Islamoglu, Timur \\
\hline Ichikawa, Masakazu & Island, J. O. \\
\hline Ichikawa, Takahiro & Itina, Tatiana E. \\
\hline Ichikawa, Yo & Ito, Shosuke \\
\hline Iemmo, Laura & Ito, Takeru \\
\hline Iervolino, Giuseppina & Ito, Takeshi \\
\hline Ignaszak, Anna & Ito, Yoshihiro \\
\hline Ignat, Maria & Itoh, Tamitake \\
\hline Ihlemann, Jürgen & Itsuno, Shinichi \\
\hline Ijima, Hiroyuki & Ivanets, Andrey \\
\hline Ijiro, Kuniharu & Ivanov, Ilia N. \\
\hline Ilahi, Bouraoui & Ivanova, Svetlana \\
\hline Ilanchezhiyan, Pugazhendi & Iwan, Agnieszka \\
\hline Ilg, Patrick & Iwanaga, Masanobu \\
\hline Illés, Balázs & Iwata, Tatsuya \\
\hline Illes, Erzsebet & Izadyar, Anahita \\
\hline Illés, Erzsébet & J. Jankiewicz, Bartłomiej \\
\hline Imamura, Gaku & Jabłońska, Beata \\
\hline Inada, Ryoji & Jacak, Jarosław \\
\hline Inagaki, Norihiro & Jacek, Żmojda \\
\hline Infantes-Molina, Antonia & Jadczak, Joanna N. \\
\hline Inoue, Shuhei & Jadwicienczak, Wojciech \\
\hline Ioannides, Theophilos & Jain, Ankur \\
\hline Ioannis, Konidakis & Jakieła, Sławomir \\
\hline Ionete, Eusebiu Ilarian & Jakšić, Olga \\
\hline Ionita, Petre & Jakubik, Wiesław P. \\
\hline Iram, Surtaj & Jakubowicz, Jarosław \\
\hline Irena, Kratochvílová & Jakubowski, Witold \\
\hline Irene Urbieta Quiroga, Ana & Jamróz, Piotr \\
\hline Irrera, Alessia & Jamting, Asa \\
\hline Irving, Tom & Janas, Dawid \\
\hline
\end{tabular}




\begin{tabular}{|c|c|}
\hline Janczarek, Marcin & Joo, Ji Bong \\
\hline Jänes, Alar & Jordan, Alex \\
\hline Jang, Changheui & Jørgensen, Bodil \\
\hline Jang, Jeong Gook & Joshi, Rakesh \\
\hline Jang, Tae-Sik & Joshi, Ravindra \\
\hline Jankauskaite, Virginija & Joshi, Sanket \\
\hline Janovák, László & Joshi, Tanmaya \\
\hline Janovec, Jozef & Jotshi, Chand \\
\hline Janowska, Izabela & Joubert, Olivier \\
\hline Jarosz-Wilkołazka, Anna & Jouini, Noureddine \\
\hline Jaszcz, Katarzyna & Ju, Heongkyu \\
\hline Javier, Sánchez-Nieves & Julien, Christian M. \\
\hline Jeeyoung, Yoo & Jun, Bong-Hyun \\
\hline Jeffryes, Clayton & Jun, Kim \\
\hline Jena, Himanshu & Jun, Young-Si \\
\hline Jeon, Ju-Won & Jung, Jaehan \\
\hline Jeon, Seokwoo & Jung, Jaehan \\
\hline Jeong, Hyung Mo & Jung, Jong Hwa \\
\hline Jeong, Jong-Ryul & Jung, Yeon-Gil \\
\hline Jeong, Nak Cheon & Jung, Yong Chae \\
\hline Jeong, Sang Moon & Juodkazis, Saulius \\
\hline Jeong, Sanghwa & Jurado Sánchez, Beatriz \\
\hline Jewell, Eifion Huw & Jurado, Amalia S. \\
\hline Jha, Birendra & Jurca, Titel \\
\hline Jha, Kshitij C. & Jursich, Gregory \\
\hline Jha, Ramesh K. & Kabanos, Themistoklis \\
\hline Jiang, Changyun & Kachynski, Aliaksandr \\
\hline Jiang, Xuchuan & Kačiulis, Saulius \\
\hline Jicsinszky, László & Kaczmarek, Anna M. \\
\hline Jimenez Ballesta, Ana Eva & Kaczorek, Ewa \\
\hline Jiménez, Juan & Kadam, Avinash \\
\hline Jiménez-Saelices, Clara & Kadkhodazadeh, Shima \\
\hline Jimenez-Villacorta, Felix & Kado, Yuya \\
\hline Jin, Hyeong Min & Kahlert, Heike \\
\hline Jing, Qingshen & Kajimoto, Shinji \\
\hline Jisha, Chandroth Pannian & Kajitani, Takashi \\
\hline Johanson, Urmas & Kako, Tetsuya \\
\hline Johansson, Christer & Kalantar-zadeh, Kourosh \\
\hline Johnson, David & Kalanur, Shankara Sharanappa \\
\hline Johnston, Linda J. & Kalas, Wojciech \\
\hline Jolly, Pawan & Kalbáčová, Marie Hubálek \\
\hline Jones, Lathe & Kaliginedi, Veerabhadrarao \\
\hline Jönsson-Niedziolka, Martin & Kalisch, Holger \\
\hline
\end{tabular}


Kallitsis, Joannis K.

Kalogirou, Orestis

Kaluža, Luděk

Kamada, Kai

Kamal, Musa R.

Kamali, Saeed

Kamanina, Natalia V.

Kameyama, Atsushi

Kamiguchi, Satoshi

Kamimura, Takafumi

Kamińska, Agnieszka

Kamitaka, Yuji

Kampouris, Dimitrios

Kanaev, Andrei

Kanazawa, Ken

Kanda, Kazuhiro

Kaneco, Satoshi

Kaneti, Yusuf Valentino

Kang, Youngsoo

Kanik, Mehmet

Kantartzis, Nikolaos V.

Kapcia, Konrad Jerzy

Kaplan, Andrey

Karagiannakis, George

Karakashev, Stoyan

Karakatsanis, Nikolaos A.

Karapanagiotis, Ioannis

Kareiva, Aivaras

Kargl, Rupert

Karle, Timothy

Karnahl, Michael

Károly, Lázár

Karousou, Eugenia

Karpov, Victor

Karpukhina, Natalia

Kartashov, Yaroslav V

Karwowska, Ewa

Kashiwagi, Shinichiro

Kasnatscheew, Johannes

Kasprowicz, Dobrosława

Katal, Reza

Kataoka, Yusuke

Katin, Konstantin P.
Kato, Haruhisa

Katsura, Shinji

Katunin, Andrzej

Kaunas, Roland

Kauppinen, Esko I.

Kaur, Amandeep

Kaushik, Nagendra

Kawabe, Yutaka

Kawakami, Hiroshi

Kawakita, Hidetaka

Kehl, Florian

Kelder, Erik M.

Keller, Adrian

Kelley, David

Kelley, Steven

Kendrick, Emma

Kerdjoudj, Halima

Ketov, Sergey

Khadka, Dhruba B.

Khalid, Ata

Khan, Omar F.

Khan, Ziaudding

Khanal, Rabi

Kharel, Parashu

Khashayar, Patricia

Khisamutdinov, Emil

Khodadoust, Amid P.

Khodaparast, Giti

Khokhlova, Marina D.

Khoo, Bee Luan

Khorasani, Sina

Khulbe, Kailash Chandra

Khyzhun, O.Yu.

Kiciński, Wojciech

Kiefer, Rudolf

Kim, Bo-Hyun

Kim, Bojeong

Kim, Bongju

Kim, Chang-Hyun

Kim, Dai-Sik

Kim, Do-Gun

Kim, Donghyun

Kim, Dongin 


\begin{tabular}{|c|}
\hline Kim, Dong-Joo \\
\hline Kim, Dong-joo \\
\hline Kim, Dongwook \\
\hline Kim, Felix \\
\hline Kim, Gue-Hyun \\
\hline Kim, Hangun \\
\hline Kim, Hyun Jae \\
\hline Kim, Hyun Tak \\
\hline Kim, Hyungwoo \\
\hline Kim, Hyunsung \\
\hline Kim, Ii Tae \\
\hline Kim, Jaeyoun \\
\hline Kim, Jeong-Chul \\
\hline Kim, Jeonghun \\
\hline Kim, Jeonghyun \\
\hline Kim, Jeongkwon \\
\hline Kim, Jong Hyun \\
\hline Kim, Jung Kyu \\
\hline Kim, Jun-Hyun \\
\hline Kim, Keun Su \\
\hline Kim, Keun-Soo \\
\hline Kim, Ki Buem \\
\hline Kim, Ki Kang \\
\hline Kim, Kyong Hon \\
\hline Kim, Moon Deock \\
\hline Kim, Moon Il \\
\hline Kim, Myungwoong \\
\hline Kim, Nam Kyeun \\
\hline Kim, Ok-Su \\
\hline Kim, Prof. Dr. Choongik \\
\hline Kim, Sang-Jae \\
\hline Kim, Seokmin \\
\hline Kim, Seong-Gon \\
\hline Kim, Seongsin \\
\hline Kim, Seunghyun \\
\hline Kim, Soo Young \\
\hline Kim, Tae Geun \\
\hline Kim, Taehoon \\
\hline Kim, Tae-Hyung \\
\hline Kim, Yoong Ahm \\
\hline Kim, Younghoon \\
\hline KIM, YUNA \\
\hline Kimata, Masafumi \\
\hline
\end{tabular}

Kioseoglou, George

Kirchner, Robert

Kirihara, Soshu

Kirm, Marco

Kirste, Ronny

Kirszensztejn, Piotr

Kiryukhantsev-Korneev, Ph.V.

Kiss, János

Kitagawa, Jiro

Kitahama, Yasutaka

Kitajou, Ayuko

Kitamura, Takayuki

Kitazawa, Takafumi

Kityk, Iwan

Klajnert-Maculewicz, Barbara

Klebert, Szilvia

Klemm, Richard

Klinkova, Anna

Kliros, George S

Kłonkowski, Andrzej M.

Kluczka, Joanna

Knibbe, Ruth

Ko, Changhyun

Ko, Dong-Kyun

Ko, Seunghwan

Kobayashi, Atsushi

Kobayashi, Jun

Kobayashi, Rika

Kobayashi, Shunsuke

Koblischka, Michael R.

Kobtsev, Sergey

Koch, Reinhold

Kochanowicz, Marcin

Koci, Kamila

Koczorowski, Tomasz

Koduru, Janardhan

Koenders, E.A.B.

Koetz, Joachim

Koh, Ahyeon

Köhler, Karsten

Köhler, Robert

Kohler, Sigmund

Kohtani, Shigeru 


\section{KOINKAR, PANKAJ}

Kojima, Toshikatsu

Kokado, Kenta

Koksharova, Olga A.

Kolanjiyil, Arun Varghese

Kolaric, Branko

Kolasinski, Kurt W.

Kolosnjaj-Tabi, Jelena

Kolpashchikov, Dmitry

Koltai, János

Konarova, Muxina

Kondo, Mio

Kong, Jaemin

Konshina, Elena

Konsolakis, Michalis

Konstantinov, Spiro M.

Kontonasaki, Eleana

Kontziampasis, Dimitrios

Koo, Hee-beom

Kooij, E. Stefan

Kooyman, Patricia

Kopecký, Dušan

Kopel, Pavel

Koplitz, Brent

Koponen, Antti

Kordas, Krisztian

Kordesch, Martin E.

Koren, Klaus

Körösi, László

Korzeniewska, Ewa

Koshimizu, Masanori

Koshizaki, Naoto

Kosinova, Marina L.

Kosinski, Pawel

Kostiainen, Mauri

Kostin, Gennadiy A.

Koszegi, Tamas

Kotek, Jan

Koter, Stanislaw

Kotsiantis, Sotiris

Kotsuchibashi, Yohei

Kottapalli, Ajay Giri Prakash

Kou, Tianyi
Koubiti, Mohammed

Koumoulos, Elias P.

Kounatidis, Ilias

Kourkoumelis, Nikolaos

Koutavarapu, Ravindranadh

Koutselas, Ioannis

Koutsogeorgis, Demosthenes

Koutzarova, Tatyana

Kouzu, Masato

Kovács, Zoltán

Kovács, Zsolt

Kovalcik, Adriana

Kovalevsky, Andrei V.

Kowalak, Stanisław

Kowalczuk, Dorota

Kowalska, Ewa

Kozak, Martin

Koziol, Mateusz

Kozliak, Evguenii I.

Kramer, Phillip

Krasnoslobodtsev, Alexey V.

Kratzer, Markus

Krause, Hans-Joachim

Krawiec, Mariusz

Kreouzis, Theo

Kriegner, Dominik

Krim, Jacqueline

Krisyuk, Vladislav

Królicka, Agnieszka

Królicka, Aleksandra

Kroll, Peter

Krstulović, Nikša

Krysinski, Pawel

Krzanowski, James

Krztoń-Maziopa, Anna

Kubo, Masataka

Kubo, Takanori

Kubota, Yoshiki

Kucherik, Alexey

Kuchler-Bopp, Sabine

Kuchmizhak, Aleksandr

Kuckling, Dirk

Kudaibergenov, Sarkyt 
Kuddannaya, Shreyas

Kudelski, Andrzej

Kudłak, Błażej

Kudlek, Edyta

Kudryashov, Sergey I.

Kujawa, Joanna

Kujawski, Wojciech

Kukli, Kaupo

Kukovec, Boris-Marko

Kukułka, Wojciech

Kulinich, Sergei

Kulinsky, Lawrence

Kulka, Michal

Kumagai, Seiji

Kumar, Anil

Kumar, Narendra

Kumar, Naresh

Kumar, Pawan

Kumar, Prasun

Kumar, Vipin

Kumar, Vivek

Kumeria, Tushar

Kumi-Diaka, James

Kunsagi-Mate, Sandor

Kuo, Chi-Ching

Kuo, Dong-Hau

Kuo, Shiaowei

Kuo, Tsung-Rong

Kupka, Teobald

Kuřitka, Ivo

Kurlyandskaya, Galina V.

Kuroda, Takashi

Kurzatkowska, Katarzyna

Kusior, Anna

Kusko, Mihaela

Kuśmierek, Krzysztof

Kustov, Leonid

Kustrowski, Piotr

Kuttner, Christian

Kuzhir, Pavel

Kuzhir, Polina P.

Kvashnin, Dmitry G.

Kvitek, Libor
Kwang, Heo

Kwon, Hyuck-In

Kwon, Ki-Young

Kwon, Nam Hee

Kwon, Oh Joong

Kwon, Oh Seok

Kwon, Soon-Hong

Kwon, Soon-Hong

Kwon, Tae-Yub

Kyzioł, Agnieszka

La Deda, Massimo

La Flor, Silvia De

La Parola, Valeria

La Spada, Luigi

Laarmann, Tim

Labuta, Jan

Lacabanne, Colette

Lacarbonara, Walter

Lachman-Senesh, Noa

Lacivita, Valentina

Lacrampe, Marie-France

Ladero, Miguel

Laforge, François O.

Lahiri, Abhishek

Lahtinen, Jouko

Lai, Chih-Hsien

Lai, Jui-Yang

Lai, Ngoc Diep

Lai, Wing-Fu

Lai, Yi Sheng

Laidani, Nadhira Bensaada

Laïk, Barbara

Laity, Peter R

Lakshminarayana, Polavarapu

Lalatonne, Yoann

Lambrecht, Walter

Lampakis, Dimitrios

Lamperska, Weronika

Lamprou, Dimitrios A.

Lan, Dongchen

Lanc, Domagoj

Lanceros-mendez, Senentxu

Lange, Holger 
Langer, Jerzy J

Lanzi, Massimiliano

Laptev, Alexander

Larese, Francesca

Larraneta, Eneko

Lartigue, Lenaic

Laskowski, Łukasz

Laso, Manuel

Latonen, Rose-Marie

Latosińska, Jolanta Natalia

Latouche, Camille

Latterini, Loredana

Lattuada, Marco

Lau, Woei Jye

Laurencin, Cato T.

Laurenti, Marco

Lavoine, Nathalie

Layek, Buddhadev

Lazzara, Giuseppe

Le Donne, Alessia

Le Ouay, Benjamin

Leavesley, David

LeBlanc, Gabriel

Lechanteur, Anna

Lechner, Bob-Dan

Leclerc, Corey

Lecomte, Philippe

Ledwon, Przemyslaw

Lee, Changwon

Lee, Che-Hsin

Lee, Chi Hwan

Lee, Chia-Hung

Lee, Chia-Hung

Lee, Dong Hyun

Lee, Dongkyu

Lee, Doojin

Lee, Eungje

Lee, Eunjung

Lee, Eunsongyi

Lee, Geunsik

Lee, Hwasung

Lee, Ilkeun

Lee, Ilkeun
Lee, Jae Sung

Lee, Jaejong

Lee, Jea Uk

Lee, Jihoon

Lee, Ju-Hyuck

Lee, Jung Seok

Lee, Kangwon

Lee, Kee-Sun

Lee, Kyung-Jin

Lee, Minbaek

Lee, Rong-Ho

Lee, Sejoon

Lee, Seok Jae

Lee, Seok Woo

Lee, Shiwoo

Lee, Taek

Lee, Woo-Kyung

Lee, Young-Chul

Leem, Jung Woo

Lefebvre, Jacques

Lefferts, Leon

Lehnherr, Dan

Leineweber, Andreas

Lemordant, Daniel

Lemus, Ranulfo

Lenaghan, Scott

Leon, Francisco

Leonardi, Francesca

Leonat, Lucia

Leong, David T.

Leonidas, Palilis

Leonyuk, N.I.

Leporatti, Stefano

Lepore, Maria

LEPRINCE-WANG, Yamin

Lerouge, Frederic

Lesch, Andreas

Lessard, Benoit

Leszczyńska, Agnieszka

Létoublon, Antoine

Lettieri, Mariateresa

Levchenko, Igor

Levesque, Pierre L. 
Levine, Mindy S.

Lewandowski, Wiktor

Lewkowski, Jaroslaw

Leżańska, Maria

Lgaz, Hassane

Lhuillier, Emmanuel

Li, Gang

Li, Jianxiong

$\mathrm{Li}$, Jiashen

Li, Meichun

Li, Oi Lun

Li, Po-Hsien

Li, Wenyue

Liakos, Ioannis

Liang, Chi-Te

Liang, Yucang

Lianos, Panagiotis

Liao, Ying-Chih

Liberato, Ferrara

Li-Destri, Giovanni

Lightfoot, Philip

Likodimos, Vlassios

Lim, Dong Chan

Lim, Eun-Kyung

Lim, Jiseok

Lim, Min-Cheol

Lim, Yee Yan

Lima, Sofia Costa

Limongi, Tania

Lin, Chia-Feng

Lin, Chu-Hsuan

Lin, Der-Yuh

Lin, Linhan

Lin, Yang-wei

Lin, Yi-Li

Lin, Yow-Jon

Lin, Yuyuan

Linares, $\mathrm{N}$.

Linko, Veikko

Liopo, Anton

Lisiecki, Aleksander

Lisiecki, Radosław

Lister, Tedd E.
Liu, Xiangsheng

Liu, Yu

Liu, Yuxin

Liverani, Liliana

Livi, Sebastien

LiVoti, Roberto

Lizundia, Erlantz

Llobet, Eduard

Llorens, José Manuel

Lo Presti, Leonardo

Löbmann, Peer

Locatelli, Andrea

Lohmus, Rynno

Lolić, Aleksandar

Lolla, Dinesh

Long, Gary J.

Longhi, Mariangela

Lopes, Maria A.

López Antón, Ricardo

López, Miguel Ángel

López-Cornejo, Pilar

Lopez-Jaramillo, Francisco Javier

López-Lorente, Ángela Inmaculada

Lopez-Manchado, Miguel Angel

López-Rayo, Sandra

López-Rubio, Amparo

Lopresti, Francesco

Lorite, Gabriela Simone

Lour, Wen-Shiung

Lourenço, João

Low, Nicholas

Lozano, R. M.

Lu, Ming-Chang

Lub, Johan

Lubal, Přemysl

Lubarda, Vlado

Luca, Oana

Lucena, Rafael

Luckham, Paul

Luduena, Richard

Ludwig, Ralf

Luisetto, Igor

Lukáč, Pavel 
Lukasik, Rafal

Lukomska-Szymanska, Monika

Lukyanov, Pavel

Luna, Carlos

Lundy, Fionnuala T

Luty, Tadeusz

Lützenkirchen, Johannes

Lützenkirchen-Hecht, Dirk

Luzi, Francesca

Ly, Thuc Hue

Lyulin, Alexey V.

Ma, Tianyi

Maas, Michael

Maasilta, Ilari

Macagnano, Antonella

Macak, Jan

Macaluso, Roberto

Maccaferri, Nicolò

Macdonald, Thomas

Machrafi, Hatim

Macoas, Ermelinda

MacRaild, Christopher

Maeda, Tomoki

Maeng, Han-Joo

Magalhães, Fernão D.

Magasinski, Alexandre

Magdanz, Veronika

Maggioni, Daniela

Magnacca, Giuliana

Magnani, Mauro

Magnucka-Blandzi, Ewa

Magnusson, Robert

Mahapatra, Manoj K.

Mahata, Manoj Kumar

Mahesh, Sachithanadam

Mahmoud, Abdelfattah

Maisano, Jessica A

Maiwa, Hiroshi

Maiz, Jon

Majak, Jüri

Mäkelä, Jyrki M.

Maki, Yasuyuki

Makkonen, Lasse
Makovec, Darko

Malandrino, Prof. Graziella

Malankowska, Anna

Malik, Aditya

Malik, Ritu

Malka, Dror

Malkin, Alexander

Mallamace, Domenico

Mallavia, Ricardo

Malvić, Tomislav

Malyi, Oleksandr I.

Mamajanov, Irena

Manakhov, Anton

Manavalan, Balachandran

Mandelli, Davide

Maneiro, Marcelino

Manera, Maria Grazia

Manfredi, Luigi

Mani, Ganesh Kumar

Manian, Avinash P.

Mankey, Gary

Mansoori, Ali

Mantanis, George

Marakatti, Vijaykumar

Marasso, Simone Luigi

Marcelli, Augusto Claudio

Marchal, Juan Antonio

Marchesan, Silvia

Marchiol, Luca

Marconcini, Paolo

Marek, Antonin

Mariani, Stefano

Marić, Milan

Mariggiò, Maria A.

Marín, Josefa Isasi

Marinkovic, Dragan

Marinkovic, Nebojsa

Marino, Attilio

Márk, Géza István

Markopoulos, Angelos

Markowska-Szczupak, Agata

Markowski, Piotr Marek

Marler, Joan 
Marmiroli, Marta

Maroni, Fabio

Marotti De Sciarra, Francesco

Marques, Carlos

Marques, Carlos

Marques, Manuel Joaquim Bastos

Marquez, Francisco M

Marradi, Marco

Marsal, Lluis F.

Martel, Bernard

Martin, Aida

Martin, Stephen M.

Martines, Emilio

Martinez Martinez, Virginia

Martinez Rodrigo, Javier

Martínez Rodrigo, Javier

Martínez, Cristina

Martínez, Elena

Martínez, Félix Quintero

Martinez-Calvo, Miguel

Martínez-Espinosa, Rosa María

Martin-Garcia, Beatriz

Martino, Marco

Martino, Sabata

Martin-Ramos, Pablo

Martins, José A.

Martins, Rodrigo

Martins, Rui C.

Martín-Sánchez, Javier

Martín-Yerga, Daniel

Martone, Alfonso

Martucciello, Nadia

Marucci, Alvaro

Maruda, Radosław W.

Masaharu, Nakayama

Masayuki, Sohgawa

Mascini, Macello

Massaro, Marina

Mastanjević, Kristina

Mastrangeli, Massimo

Masuda, Yoshitake

Masuo, Sadahiro

Matei, Andreea
Materer, Nicholas F.

Mathew, Nithin

Mathieu, Didier

Matras-Postolek, Katarzyna

Matsuhisa, Naoji

Matsui, Isao

Matsumoto, Kozo

Matsuno, Tomonori

Matsuo, Takashi

Matsushima, Hisayoshi

Matsuura, Tomoaki

Matwijczuk, Arkadiusz

Matykiewicz, Danuta

Maupin-Furlow, Julie A.

Mauriello, Francesco

Mautner, Andreas

Mavrogonatou, Eleni

Mayer, Dirk

Mayer, Thomas

Mayr, Peter

Mazur, Maciej

Mba Blázquez, Miriam

McCarty, Owen J.T.

McDonald, Thomas

McGilly, Leo J.

Medronho, Bruno Filipe Figueiras

Megiel, Elżbieta

Mehta, Jodhbir

Mei, Bastian

Meijide, Francisco

Meilhan, Jérome

Meixner, Alfred J.

Mekonnen, Tizazu

Melchionna, Michele

Mele, Elisa

Melin, Frederic

Mélinon, Patrice

Meloni, Eugenio

Memoli, Gianluca

Menaszek, Elżbieta

Méndez, Bianchi

Menegazzo, Federica

Meneghetti, Giovanni 
Menendez, Beatriz

Menendez, Miguel

Menger, Fredric M

Menini, Maria

Menon, Jyothi

Meo, Michele

Meo, Paolo Lo

Merabia, Samy

Mercado, Candy C

Mercone, Silvana

Merle, Benoit

Meroni, Daniela

Meskinis, Sarunas

Meškys, Rolandas

Messina, Fabrizio

Mestre, Ana S.

Mestres, Narcís

Meulenberg, Robert W.

Meyyappan, Meyya

Meziani, Mohammed

Mezzi, Alessio

Miah, Abdul Halim

Miccio, Francesco

Michail, Christos

Michalik, Jan

Michalkiewicz, Beata

Michel, Carlos R.

Michna, Aneta

Miculescu, Florin

Miecznikowski, Krzysztof

Miederer, Matthias

Miglietta, Maria Lucia

Mikhelson, Konstantin

Milanesio, Marco

Milano, Francesco

Milano, Gianluca

Milasius, Rimvydas

Milčius, Darius

Milionis, Athanasios

Miluski, Piotr

Minak, Giangiacomo

Minakshi, Manickam

Minami, Ichiro
Minea, Alina Adriana

Mineva, Tzonka

Mínguez, Rebeca De Nalda

Minin, Igor V.

Miranda-Castro, Rebeca

Miseki, Yugo

Mishra, Rupesh K.

Mishra, Yogendra

Misyura, Sergey Y.

Mitchell, Andrew

Mitchell, John

Mitchenko, Serge

Mitomo, Hideyuki

Mitran, Sorin

Mitropoulos, Athanasios C.

Mittova, Irina Ya

Miyazaki, Koji

Mizoshita, Norihiro

Mizsei, János

Mo, Kun

Mobbili, Giovanna

Modica, Maria

Moerman, David

Moghimi, SM

Mohanta, Antaryami

Mohd-Yasin, Faisal

Mohles, Volker

Moineau-Chane-Ching, Kathleen I.

Moita, Ana Sofia

Molina, C.B.

Moliner Martinez, Yolanda

Molla, Md. Ashraful Islam Molla

Molle, Alessandro

Moloney, Mark G.

Momeni, Kasra

Momida, Hiroyoshi

Monai, Matteo

Mondal, Sudip

Monfort, Olivier

Monteiro Baptista, António Paulo

Montgomery, Jason M.

Monti, Alessio

Monticelli, Orietta 
Monzón, Antonio

Mooers, Blaine

Moosmann, Julian

Morais, Simone

Morales, Julián

Moralli, Daniela

Morant, Carmen

Morasch, Alexander

Morasso, Carlo

Morávková, Zuzana

Morazzoni, Franca

Morbidelli, Massimo

Moreau, Julien

Moreno, Fernando

Moreno, Inés

Moreno, Miguel

Moretti, Elisa

Morganti, Pierfrancesco

Mori, Wasuke

Morimoto, Nobuyuki

Morita, Masamune

Morkoc, Hadis

Morley, Nicola A

Moroni, Giovanni

Morozan, Adina

Morris, Jeffrey F.

Mortazavi, Bohayra

Moscone, Danila

Mosk, Allard

Motobayashi, Kenta

Mott, Derrick M.

Mourdikoudis, Stefanos

Mouzakis, Dionysios E.

Mozia, Sylwia

Mrázek, Jan

Mrlík, Miroslav

Mrówczyński, Radosław

Mróz, Waldemar

Mróz, Zenon

Mrukiewicz, Mateusz

Mucalo, Michael R.

Mucsi, Zoltán

Muench, Falk
Muenzenberg, Markus

Muguruma, Hitoshi

Mukherjee, Soumya

Mukherjee, Sudip

Müller, Bert

Müller, Danny

Muller, Gerhard

Müller, Gerhard

Müller, Karsten

Mulmudi, Hemant Kumar

Muniz-Miranda, Francesco

Muniz-Miranda, Maurizio

Muñoz, Macarena

Muñoz-Batista, Mario Jesús

Munteanu, Florentina-Daniela

Murase, Norio

Muravyev, Nikita V.

Murcia-López, Sebastián

Musić, Svetozar

Musmarra, Dino

Muszyński, Tomasz

Muziol, Tadeusz Mikolaj

Myśliwiec, Jarosław

$\mathrm{Na}$, Kyungsu

Nabae, Yuta

Nadolny, Zbigniew

Nagai, Masaya

Nagai, Noriaki

Nagao, Daisuke

Nagatani, Naoki

Nagy, Dénes Lajos

Nagy, Endre

Nair, Ashwin

Nair, Devatha P.

Nair, Sandeep Sudhakaran

Naito, Katsuyuki

Najlah, Mohammad

Nakagawa, Yoshinao

Nakahara, Yoshio

Nakamura, Daisuke

Nakashima, Seiji

Nalbach, Peter

Namieśnik, Jacek 
Nanaki, Stavroula

Nanver, Lis K.

Narciso, Javier

Nasiri, Noushin

Nataraj, Latha

Nattestad, Andrew

Naumov, Anton

Naumova, Ella A.

Naushad, M

Navalon, Sergio

Navarro, Rodrigo

Nayak, Arunima

Neagu, Adrian

Neal, Luke

Neel, Ensanya A. Abou

Nees, Matthias

Neffe, Axel T.

Negrete, George

Negro, Carlos

Neipp, Cristian

Nemes, Norbert M.

Nemestothy, Nandor

Németh, Péter

Nemnes, George Alexandru

Neri, Giovanni

Nesterov, Dmytro

Nesterova, Oksana V.

Neugebauer, Dorota

Nevshupa, Roman A

Ngamchuea, Kamonwad

Ngene, Peter

Nguyen, Duc Thanh

Nguyen, Nhat Truong

Nguyen-Tri, Phuong

$\mathrm{Ni}$, Guangxin

$\mathrm{Ni}$, Melody

Niaura, Gediminas

Nicholson, John W.

Nicoletta, Fiore Pasquale

Niemi, Tapio

Niemirowicz-Laskowska, Katarzyna

Nien, Yung-Tang

Nienborg, Björn
Nieszporek, Krzysztof

Nieto, Andy

Niewa, Rainer

Niidome, Takuro

Nikolelis, Dimitrios P.

Nikumb, Suwas

Nilges, Tom

Nima, Zeid A.

Nishikiori, Hiromasa

Nishiyabu, Ryuhei

Nisnevitch, Marina

Nistor, Ileana Denisa

Nitschke, Mirko

Nobili, Andrea

Nocentini, Sara

Nofz, Marianne

Nogueira, Helena

Nogues, Josep

Nomoto, Takahiro

Nomura, Shintaro

Nonomura, Dr. Kazuteru

Norek, Małgorzata

Norton, Michael

Noubactep, Chicgoua

Nouranian, Sasan

Novikov, Andrei

Novio, Fernando

Novotna, Vladimira

Nowak, Sascha

Nozomi, Takeuchi

Ntalii, Nikoletta

Ntalli, Nikoletta G.

Nunes, Carla D.

Nunes, Daniela

Núñez Carmona, Estefanía

Nurunnabi, Md

Nypelo, Tiina

O'Mullane, Anthony

O'Rear, Edgar

Obata, Yasuko

Obraztsov, Alexander N.

O'Brien, Casey P.

Ochi, Masayuki 
Ochiai, Bungo

Ochiai, Tsuyoshi

Ocłoń, Paweł

O'Connor, Brian

Oćwieja, Magdalena

O'Duill, Sean

Ogasawara, Hirohito

Ogasawara, Toru

Ogawa, Shinpei

Ogino, Isao

Oh, Jonghyun

Ohara, Keishi

Ohashi, Masashi

Ohlckers, Per

Ohsedo, Yutaka

Okada, Narihito

Okamoto, Hideki

Okamoto, Hiroyuki

Okamoto, Masami

Okazaki, Joji

Okazaki, Toshiya

Okhrimchuk, Andrey

Okochi, Mina

Olchowka, Jacob

Oliveira, Mariana Braga

Oliveira, Paula A.

Oliveira, Vítor

Ollila, Samuli

Olmos, Dania

Olsen, Lars Folke

Olthuis, Wouter

Omastová, Mária

Ono, Atsushi

Opitz, Jörg

Orbea, Amaia

Oreshonkov, Aleksandr

Orge, Carla

Orive, Alejandro González

Orman, Mehmet

Ormeci, Alim

Orobtchouk, Regis

Ortega, José Marcos

Ortenzi, Marco A.
Ortiz, Alfredo

Ortolani, Luca

Osaki, Tomohiro

Oschatz, Martin

Osipov, Andrey

Osipov, Vladimir

Osses, Nelson

Ostasevicius, Vytautas

Ostrauskaite, Jolita

Osvath, Zoltan

Osvet, Andres

Ota, Satoshi

Otero, Marta

$\mathrm{Ou}$, Jianzhen

Ouerghi, Abdelkarim

Ouyang, Zi

Oya, Yuichi

Oyama, Kotaro

Pacheco Tanaka, David Alfredo

Paciorek-Sadowska, Joanna

Packirisamy, Muthukumaran

Pacurari, Maricica

Paczesny, Jan

Padalkar, Sonal

Padamati, Ramesh Babu

Padil, Vinod V. T.

Padnya, Pavel

Pagano, Stefano

Pagès, Stéphane

Pagliara, Stefania

Paik, Taejong

Pal, Sudipto

Palamà, Ilaria

Palchoudhury, Soubantika

Palermo, Edmund

Palinko, Istvan

Pałka, Norbert

Palkowski, Heinz

Pallandre, Antoine

Pallaoro, Alessia

Palma, Luca

Palma, Paulo

Palmieri, Valentina 
Palomares, Antonio Eduardo

Palomba, Valeria

Palumbo, Fabio

Palzer, Stefan

Pamies, Ramon

Pana, Ovidiu

Panagopoulos, C. N.

Pandey, Ravi

Pané Vidal, Salvador

Panek, Piotr

Panek, Rafal

Panikkanvalappil, Sajanlal R.

Panomsuwan, Gasidit

Panzarasa, Guido

Paolicelli, Guido

Pap, József

Papadimitriou, Vassiliki

Papadopoulos, Antonios N.

Papageorgiou, George Z.

Papanikolaou, Nikolaos

Papavasiliou, Joan

Papavassiliou, Dimitrios

Papini, Emanuele

Papoulis, Dimitris

Pappas, George S.

Parajuli, Durga

Parakhonskiy, Bogdan

Paraskevopoulou, Patrina

Pardanaud, Cédric

Parigger, Christian

Parisi, Filippo

Parisini, Emilio

Park, Byung-Wook

Park, Chul-ho

Park, Daehwan

Park, Deog-Su

Park, Deok-Yong

Park, Dong Hyuk

Park, Enoch

Park, Jaehoon

Park, Jin Gyu

Park, Jin Kuen

Park, Joo-Cheol
Park, Jung-hyun

Park, Junyong

Park, Kwi-Il

Park, Kwi-Il

Park, Kyeongsoon

Park, Mira

Park, Myung Hwan

Park, SaeJune

Park, Sangmoon

Park, Sunggook

Park, Sungha

Park, Wansu

Park, Young Wook

Parkinson, Dilworth

Parlea, Lorena

Parlett, Christopher

Parlińska-Wojtan, Magdalena

Parmentier, Julien

Parnell, Andrew J.

Parnian, Mohammad Javad

Parsons, J.G.

Parvez, Khaled

Pascual, Laura

Pascuta, Petru

Pasini, Dario

Pasinszki, Tibor

Pasquinelli, Gianandrea

Passalacqua, Rosalba

Pastore, Roberto

Pastrana-Martínez, Luisa M.

Patanè, Salvatore

Patelli, Alessandro

Pathakoti, Kavitha

Patil, Santosh S

Pauliac-Vaujour, Emmanuelle

Pauliukaite, Rasa

Paulsen, Hauke

Pavesi, Maura

Pavlatou, Evangelia A.

Pavlos, Klepetsanis

Pawar, Radheshyam

Pawar, Shital

Pawlak, Michał 
Pawłat, Joanna

Pawlus, Paweł

Peale, Robert

Pedraza, Luís Cerdán

Pedro Acuña, Guillermo

Pegoretti, Alessandro

Peijnenburg, Willie J. G. M.

Pelin, Marco

Peljo, Pekka

Pelka, Rafał

Pellegrino, Francesco

Pelli, Stefano

Peña, Jose Ignacio

Peng, Ruogu

Peng, Zhili

Penkov, Oleksiy V.

Pensabene, Virginia

Peppas, Georgios

Peppel, Tim

Perche, Federico

Pereira Gonçalves, António

Pereira, Cláudia C. L.

Pereira, Eulalia

Pereira, Luís

Pereira, Luiz Fernando Ribeiro

Peres, José A.

Pérez Comunas, María José

Perez Del Pino, Angel

Pérez Menéndez, Ramón José

Pérez-Álvarez, Leyre

Pérez-Juste, Ignacio

Pérez-Mendoza, Manuel J.

Pérez-Prieto, Julia

Périgo, Élio Alberto

Perinelli, Diego Romano

Peris, José-Esteban

Perkins, Laura E. L.

Perlepes, Spyros P.

Perles, Josefina

Perrotti, Vittoria

Perumal, Suguna

Peshek, Timothy

Pestov, Alexander
Petala, Natasa

Petersen, Elijah

Petersen, Eric

Peterson, Dominic S.

Petkov, Nikolay

Petrella, Andrea

Petrič, Marko

Petrila, Iulian

Petrò, Stefano

Petrova, Krasimira T.

Petrovic, Zeljka

Petrovykh, Dmitri Y

Petukhov, Andrei V.

Peveler, William

Philipose, Usha

Phillips, Jonathan

Phillips, Laurie

Phoenix, S. Leigh

Piasecki, Adam

Pica, Monica

Piccirillo, Clara

Pichon, Céline

Pieczka, Adam

Pielichowska, Kinga

Pielichowski, Krzysztof

Piergiovanni, Luciano

Pierini, Filippo

Pietrasik, Joanna

Pifferi, Valentina

Pikulin, Alexander

Pilot, Roberto

Pina, João

Pina, Sandra

Pineda Rodríguez, Teresa

Pineda, Antonio

Pineda, Eloi

Pineiro, Manuel M.

Pingitore, Nicholas

Pinho, Luís

Pini, Valerio

Pinkas, Iddo

Pinto Da Silva, Luís

Pinto, Nicola 
Piozzi, Antonella

Piperno, Anna

Piperopoulos, Elpida

Piro, Benoit

Pirri, Angela

Piscitelli, Filomena

Pispas, Stergios

Pistone, Alessandro

Piszczek, Piotr

Pita, Marcos

Pittalà, Valeria

Pitto-Barry, Anaïs

Pixley, Sarah

Pizzoferrato, Roberto

Plonska-Brzezinska, Marta

Plotniece, Aiva

Plyushch, Artyom

Pochard, Isabelle

Pochat-Bohatier, C.

Podlipná, Radka

Pohl, Pawel

Poitras, Daniel

Pokharel, Pashupati

Pokhrel, Madhab

Polat, Emre Ozan

Polavarapu, Lakshminarayana

Polimeni, Antonella

Polimeno, Antonino

Politano, Antonio

Polito, Laura

Polowczyk, Izabela

Polyakov, Nikolay

Poma, Alessandro

Poma, Anna

Pomastowski, Paweł Piotr

Pons, Josefina

Pons, Thomas

Ponzoni, Andrea

Popa, Lacramioara

Popielarz, Roman

Porat, Ze'ev

Porrati, Fabrizio

Porwal, Harshit
Postigo, Pablo Aitor

Postnikov, Pavel

Potapov, Andrei

Poumirol, Jean-Marie

Pourceau, Gwladys

Pourrahimi, Amir Masoud

Pourret, Olivier

Pourrezaei, Kambiz

Povolotskiy, Alexey V.

Poyato, Rosalía

Pradanos, Pedro

Pradhan, Dhiren K.

Pradhan, Sangram K.

Prado-Gotor, Rafael

Prakash Kottapalli, Ajay Giri

Prassl, Ruth

Prat, Maria

Praus, Petr

Predoi, Daniela

Prida, Victor M.

Prieto, Carlos

Prieto, Cristina

Printz, Adam D.

Prochazka, Marek

Prochowicz, Daniel

Profire, Lenuta

Prokopidis, Konstantinos

Prokopowicz, Magdalena

Prudenzano, Francesco

Pruna, Alina Iuliana

Prytz, Øystein

Ptok, Andrzej

$\mathrm{Pu}$, Ying-Chih

Pucci, Andrea

Puga, Alberto V.

Puglia, Debora

Puglisi, Rosaria

Puiggalí, Jordi

Puli, Venkata Sreenivas

Pullano, Salvatore

Pum, Dietmar

Punzo, Francesco

Puppi, Dr. Dario 
Purcar, Violeta

Puszkiel, Julián

Putkonen, Matti

Putla, Sudarsanam

Putz, Mihai V.

Pyatenko, Alexander

Pyrgioti, Eleftheria

Qiang, Zhe

Quagliariello, Vincenzo

Quang Trung, Tran

Quémerais, Bernadette

Quesne, Matthew

Quijada-Garrido, Isabel

Quintanilla, Asuncion

Quinto, Emiliano José

Quynh Hoa, Le

Raabe, Gabriele

Rabczuk, Timon

Raccichini, Rinaldo

Rack, Philip D.

Radadia, Adarsh D.

Radhakrishnan, Sivaprakasam

Radtke, Aleksandra

Radziuk, Darya

Rafiee, Mohammad

Raga, Sonia R.

Ragab, Tarek

Raganati, Federica

Ragona, Laura

Ragusa, Andrea

Rahimi, Parvaneh

Rahman, Md. Mahbubur

Rahme, Kamil

Rai, Amritesh

Rai, Prakash

Rainer, Alberto

Rajapandiyan, Panneerselvam

Rajendran, Jagathesh Chandra

Ramakumar, Kinthada

Ramanavicius, Arunas

Ramapuram, Jay

Ramirez-Castellanos, Julio

Ramirez-Vick, Jaime
Ramos Cabrer, Pedro

Ramos, Daniel

Ranc, Václav

Ranjit, Suman

Ranjkesh, Amid

Rankin, Rees B.

Ranoszek-Soliwoda, Katarzyna

Ranzato, Elia

Raposo, Maria

Rasheed, Tahir

Rashid, Mohammad Mamunur

Raspanti, Mario

Ratnakumar, B. V.

Raty, Jean-Yves Raty

Raucci, Maria Grazia

Raudino, Antonio

Rault-Berthelot, Joëlle

Rauwel, Erwan

Rauwel, Protima

Ravelli, Davide

Ravnsbæk, Dorthe B.

Ravula, Sudhir

Razdolski, Ilya E.

Read, Jeffrey

Reber, Christian

Rebollar, Esther

Reddy, M. Venkatashamy

Reeves-McLaren, Nik

Rego, Gaspar

Regulska, Elzbieta

Reimhult, Erik

Reinhard, Friedemann

Reinosa, Julián Jiménez

Reipa, Vytas

Reis, Paulo Nobre Balbis Dos

Rej, Sourav

Reli, Martin

Remes, Mgr. Zdenek

Rene, Eldon R.

Renman, Gunno

Rennhofer, Harald

Renò, Filippo

Rerat, Michel 
Restivo, João

Reukov, Vladimir

Reverberi, Andrea P.

Reyes-Ortega, Felisa

Rhee, Jong Il

Ribeiro, Clarisse

Ribeiro, Daniela

Ribis, Joel

Riccardo, Carlo

Ricci, Marilena

Riccò, Raffaele

Richard, Cyrille

Richard, Mandle

Richardson, Joseph

Richtera, Lukáš

Rider, Andrew N.

Ridi, Francesca

Riedl, Bernard

Riela, Serena

Riess, Gisbert

Riha, Shannon C.

Rim, Kyung Taek

Rim, You Seong

Rimondini, Lia

Rinkevich, Anatoly

Rioual, $S$.

Rivadeneyra, Almudena

Riveiro, Antonio

Rizal, Conrad

Riziotis, Christos

Rizzi, Gian Andrea

Rizzo, Antonio

Rizzo, Carla

Rizzolio, Flavio

Roberts, Nicholas

Robinson, Donald A.

Rocai Cabarrocas, Pere

Rocchitta, Gaia

Rodrigo, Sergio G

Rodríguez Bernaldo De Quirós, Ana

Rodriguez Diaz, Camilo Arturo

Rodriguez, Javier

Rodriguez, Noel
Rodríguez, Rafael

Rodríguez-Jerez, José Juan

Rodríguez-López, Joaquín

Rodriguez-Lorenzo, Laura

Rogala, Maciej

Roh, Changhyun

Rojo, Luis

Romanato, Filippo

Roma-Rodrigues, Catarina

Romeo, Francesco

Romero, Francisco J.

Romero, Isabel

Romero-Enrique, Jose M.

Romero-Salguero, Francisco J.

Roppolo, Ignazio

Rosado, Catarina

Rossella, Francesco

Rossetti, Ilenia

Rostovtsev, Yuri

Rouhani, Shamileh

Rowe, Alistair

Roy, Anupam

Roy, Sagar

Rozga, Pawel

Rozynek, Zbigniew

Rtimi, Sami

Rubino, Federico Maria

Ruda (FRSC), Harry

Rudzinski, Joseph

Rudziński, Wojciech

Ruffino, Francesco

Ruggieri, Fabrizio

Ruggiero, Alessandro

Ruiz De Larramendi, Idoia

Ruman, Tomasz

Rumpf, Klemens

Rumyantseva, Marina

Rurali, Riccardo

Russell, Thomas

Russo, Patrícia A.

Russo, Pietro

Russo, Saverio

Russo, Teresa 
Ruths, Marina

Ryabchikova, Elena I.

Ryan, James G.

Rybak, Andrzej

Rybiński, Przemysław

Rydosz, Artur

Rydz, Joanna

Ryu, Ja-Hyoung

Ryu, Jehwang

S. Oh, Daniel

Sabirov, Denis

Saccà, Ada

Sacco, Adriano

Sacco, Olga

Sada, Kazuki

Sadowska, Beata

Sadreev, Almas F.

Safa, Kasap

Safaei, Mohammad Reza

Safdari Shadloo, Mostafa

Safiuddin, Md.

Sagué, Anna Laromaine

Saha, Gobinda

Sahoo, Sumantha

Sahu, Saura C.

Saielli, Giacomo

Saijo, Yoshifumi

Saini, Naurang

Saito, Genki

Saito, Noriko

Saive, Rebecca

Sakaebe, Hikari

Sakai, Hiromi

Sakai, Joe

Sakar, Mohan

Sakthivel, Tamilselvan

Sala, Roberto

Salahub, Dennis

Salaoru, Iulia

Salas, Carlos

Salguero, Tina T.

Salinas Rodríguez, Beatriz

Salleras, Marc
Salmas, Constantinos

Samad, Yarjan Abdul

Samanta, Pralok Kumar

Samaras, Ioannis

Samoila, P. Mugurel

Samokhvalov, Alexander

Samuel, Temesgen

Samulionis, Vytautas

San Fabian, Emilio

Sanchez, Goar

Sánchez-García, David

Sánchez-Royo, Juan Francisco

Sánchez-Tovar, Rita

Sancini, Giulio

Sandanayaka, Atula S. D.

Sandre, Oliver

Sandri, Giuseppina

Sandulescu, Madalina

Sangiovanni, Davide

Sankar, Meenakshisundaram

Sankaran, Kamatchi Jothiramalingam

Sannino, Diana

Sansone, Anna

Sansone, Lucia

Santamaria, Rita

Santangelo, Saveria

Santer, Svetlana

Santhanam, KSV

Santoni, Antonino

Santoni, Marie-Pierre

Santos, Abel

Santos, Elizabeth

Santulli, Carlo

Sanz-Garcia, Andres

Saoud, Khaled

Sapi, Andras

Sarantopoulou, Evangelia

Sarasini, Fabrizio

Sarker, Dipak

Sarker, Subrata

Sarlin, Essi

Sarma, Raktim

Sarris, Ioannis 


\begin{tabular}{|c|c|}
\hline Sasai, Yasushi & Sciortino, Alice \\
\hline Sasaki, Tomoyuki & Sciortino, Luisa \\
\hline Sashiwa, Hitoshi & Scoponi, Marco \\
\hline Šatínský, Dalibor & Scotognella, Francesco \\
\hline Sato, Kazunori & Scott, Amy M. \\
\hline Sato, Kimiyasu & Scrimin, Paolo \\
\hline Satriano, Cristina & Secenji, Aleksandar \\
\hline Saturnino, Carmela & Secu, Elisabeta-Corina \\
\hline Savelyeva, Natalia & Secu, M. \\
\hline Sawada, Hideaki & Sedlačík, Michal \\
\hline Sawada, Toshiki & Segawa, Hiroyo \\
\hline Sberveglieri, Veronica & Seifi-Mofarah, Sajjad \\
\hline Scaffaro, Roberto & Seisenbaeva, Gulaim A. \\
\hline Scaini, Denis & Seitz, W. Rudolf \\
\hline Scarano, Domenica & Sekatski, Serguei \\
\hline Scardaci, Vittorio & SEKINE, Tomohito \\
\hline Scardi, Paolo & Sel, Ozlem \\
\hline Scarpa, Marina & Selimefendigil, Fatih \\
\hline Scarso, Alessandro & Selke, Matthias \\
\hline Scendo, Mieczyslaw & Semenov, Konstantin \\
\hline Schaefer, Jennifer & Semisalova, Anna \\
\hline Schäfer, Helmut & Sengupta, Bidisha \\
\hline Scheicher, Ralph H. & Seo, Hyung Kee \\
\hline Schepler, Kenneth & Sequeira, César A.C. \\
\hline Scherf, Ullrich & Serchi, Tommaso \\
\hline Schilirò, Emanuela & Sergey, Makarov \\
\hline Schintke, Silvia & Serpooshan, Vahid \\
\hline Schirhagl, Romana & Serrà, Albert \\
\hline Schlater, Guy & Serrano-Aroca, Ángel \\
\hline Schmid, Markus & Sessolo, Michele \\
\hline Schmidbaur, Hubert & Seynhaeve, Ann L. B. \\
\hline Schmidt, Bernhard & Shabatina, Tatyana \\
\hline Schmitt, Katrin & Shacham-Diamand, Yosi \\
\hline Schmutz, Marc & Shadike, Zulipiya \\
\hline Schnabel, Thomas & Shafiei, Mahnaz \\
\hline Schneider, Raphael & Shah, Furqan A. \\
\hline Scholle, Markus & Shahjamali, Mohammad \\
\hline Scholz, Ferdinand & Shallcross, Sam \\
\hline Schueneman, Greg & Sham, T.K. \\
\hline Schulz, Florian & Shams, S. Fatemeh \\
\hline Schulz, Sebastian Andreas & Shang, Luoran \\
\hline Schulze, Margit & Shankar, Eswar \\
\hline Schweitzer, Dietrich & Shapiro, Bruce \\
\hline
\end{tabular}


Shapovalov, Victor

Shapter, Joe

Sharifi, Tiva

Sharkeev, Yurii

Sharma, Anirudh

Sharma, Piyush Sindhu

Sharma, Saumya

Sharma, Swati

Shaw- Stewart, James

Shayduk, Roman

Shaygan, Mehrdad

Shcherbakov, Maxim R.

Shchyglo, Oleg

Shearer, Cameron J.

Shehata, Nader

Shehu, Rafael

Sheka, Elena F.

Shekargoftar, Masoud

Shellaiah, Muthaiah

Shenashen, Mohamed A.

Shenderovich, Ilya G.

Sher, Chin-Wei

Sheremet, Mikhail

Sheri, Madhu

Sherrell, Peter

Shestakov, Mikhail

Shi, Zengqian

Shields IV, C. Wyatt

Shilova, Olga

Shim, Junho

Shimada, Toshihiro

Shimanouchi, Toshinori

Shimizu, Flavio

Shimizu, Kazuo

Shimobayashi, Shunsuke F.

Shimoi, Norihiro

Shimojo, Masayuki

Shimomura, Osamu

Shimomura, Takeshi

Shin, Bo-Sung

Shin, Su Ryon

Shinde, Sudhirkumar

Shirokov, Vladimir B.
Shokouhimehr, Mohammadreza

Shpacovitch, Victoria

Shrestha, Lok

Shtepliuk, Ivan

Sibillano, Teresa

Siciliani, Mario

Sideratou, Zili

Sidorenko, Alexander

Siegel, Jakub

Šiffalović, Peter

Sigle, Wilfried

Signore, Giovanni

Sikora, Jan

Sikora, Paweł

Silaghi-Dumitrescu, Radu

Silikas, Nikolaos

Silva, Filomena

Silva, Maria Joao

Silva, Teresa Moura E

Silvain, Jean-François

Silvan, Miguel Manso

Silveira, Célia

Silver, Jack

Silvestre, Samuel

Sim, Uk

Sima, Felix

Simeonidis, Konstantinos

Simha Martynkova, Grazyna

Simion, Cristian E.

Simmonds, Paul J.

Simões, Sónia

Simon, Sebastien

Simonescu, Claudia Maria

Simpson, David A.

Simserides, Constantinos

Singh, Ashok K.

Singh, David

Singh, Gautam

Singh, Gurwinder

Singh, Jai

Singh, Seema

Singha, Subhankar

Sinha Ray, Sumit 


\author{
Sinha, Jasmine \\ Sinha, Sudarson \\ Sinha-Ray, Suman \\ Siniscalco, Dario \\ Sinn, Gerhard \\ Siracusa, Valentina \\ Sirkar, Kamalesh \\ Sirutkaitis, Valdas \\ Sisti, Laura \\ Sitarz, Maciej \\ Siwińska-Ciesielczyk, Katarzyna \\ Skorik, Yury A. \\ Skowroński, Łukasz \\ Skwarek, Ewa \\ Ślebarski, Andrzej \\ Sloan, Jeremy \\ Slobodian, Petr \\ Sloma, Marcin \\ Słomkowski, Stanislaw \\ Smani, Younes \\ Smått, Jan-Henrik \\ Smith Callahan, Laura A. \\ Smith, Adam \\ Smith, Bryan \\ Smith, Henry I. \\ Smith, Robert E. \\ Smolyanitsky, Alex \\ Snee, Preston \\ Šob, Mojmír \\ Sobolev, Konstantin \\ Sobotta, Lukasz \\ Socol, Marcela \\ Sogorb Sánchez, Miguel Ángel \\ Sohn, Youngku \\ Sola, Ramona \\ Soldano, Caterina \\ Solomonov, Alexey V. \\ Son, Donghee \\ Son, Moon \\ Son, Seung Uk \\ Sonde, Sushant \\ Søndergaard, Thomas \\ Song, Sungho
}

Song, Young Min

Soon, Jong Jeong

Sorrentino, Andrea

Sotgiu, Giovanni

Sotiriou-Leventis, Chariklia

Sotiropoulos, Sotiris

Soto, Paul

Sotome, Masato

Sowa, Ireneusz

Spagnuolo, Gianrico

Spanopoulos, Ioannis

Speghini, Adolfo

Speranzini, Emanuela

Spieß, Lothar

Spirk, Stefan

Sportelli, Maria Chiara

Sprio, Simone

Sprynskyy, Myroslav

Srinivas, Keerthi

Srivastava, G.P.

St John, James A.

Stadnichenko, Andrey

Stafeev, Sergey

Stafford, Luc

Stahl, Frank

Stamate, Eugen

Stamatis, Haralambos

Stan, Miruna

Stanca, Sarmiza Elena

Stańczyk, Wlodzimierz A.

Staneva, Desislava

Staruch, Margo

Stassen, Ivo

Staszak, Katarzyna

Stavenga, Doekele G.

Stedman, Kenneth

Stefan, Neatu

Stefanelli, Manuela

Štefanić, Petra Peharec

Stegmann, Philipp

Steinbach-Rankins, Jill

Steinfeldt, Norbert

Steinhauer, Stephan 
Stellato, Francesco

Stelmachowski, Paweł

Stepanov, Gennady V.

Stephan, Holger

Stępniak, Izabela

Stępniowski, Wojciech

Steup, Martin

Stewart, George C.

Stievano, Lorenzo

Stine, Keith J.

Stinespring, Charter

Stiufiuc, Rares

Stobiecka, Magdalena

Stöckelhuber, Klaus Werner

Stoikov, Ivan Ivanovich

Stojek, Zbigniew

Stolarczyk, Agnieszka

Stoleru, Elena

Stolojan, Vlad

Strankowski, Michal

Strelniker, Yakov

Stride, John Arron

Strini, Alberto

Strunk, Jennifer

Strzelecka-Kiliszek, Agnieszka

Stylianakis, Minas M.

$\mathrm{Su}, \mathrm{Chia-Hao}$

$\mathrm{Su}$, Wen-Ta

Suárez, Isaac

Suarez, Sebastian

Suber, Lorenza

Suckeveriene, Ran

Sugai, Yuichi

Sugawa, Kosuke

Sugiura, Yuki

Suh, Joonki

Sukhov, Sergey

Sumboja, Afriyanti

Sun, An-Cheng

Sun, Conroy

Sun, Hao

Sun, Hongyu

Sun, Wujin
Sun, Xiangcheng

Sundqvist, Jonas

Sunna, Anwar

Surmenev, Roman A.

Suryanto, Bryan

Suzuki, Shinya

Suzuki, Yoshio

Suzuki, Yuki

Svarnas, Panagiotis

Svensson, Ann Mari

Svorc, Lubomir

Swaaij, René Van

Sweetman, Martin

Sykora, Milan

Syrovy, Tomas

Szabó, Tamás

Szałowski, Karol

Szczerba, Jacek

Szczurek, Andrzej

Szekely, Gyorgy

Szepvolgyi, Janos

Szewczyk, Roman

Szilágyi, Imre Miklós

Szilvási, Tibor

Szőri, Milán

Szperlich, Piotr

Szukalski, Adam

Szybowicz, Miroslaw

Szymańska, Renata

Szymborski, Tomasz

Szymczyk, Anna

Tabarraei, Alireza

Taboryski, Rafael J.

Tadanaga, Kiyoharu

Tagliatesta, Pietro

Taglietti, Angelo Maria

Tahergorabi, Reza

Taheri, Shima

Tai, Cheuk-Wai

Tailhades, Philippe

Takafuji, Makoto

Takagaki, Atsushi

Takahashi, Kazuyuki 
Takahashi, Keisuke

Takahashi, Yasuo

Takahiro, Katsumi

Takamatsu, Seiichi

Takano, Toshiyuki

Takashima, Toshihiro

Takayoshi, Suzuki

Takeoka, Shinji

Takeshita, Fumitaka

Takeshita, Satoru

Takeuchi, Tomonari

Talaat, Ahmed

Talapaneni, Siddulunaidu

Taleb, Abdelhafed

Tallarico, Marco

Tampucci, Silvia

Tamulevičienè, Asta

Tanabe, Katsuaki

Tanaka, Shinji

Tanaka, Tomonari

Tangoulis, Vassilis

Tanikawa, Tomoyuki

Tański, Tomasz

Tao, Shanwen

Tappertzhofen, Stefan

Taratula, Olena

Tard, Cédric

Tardy, Blaise

Tasca, Federico

Tasis, Dimitrios

Tateishi-Karimata, Hisae

Tatullo, Marco

Tavakoli, Javad

Tavares, Anthony

Tayabali, Azam

Tayade, Rajesh

Tayeb, Ali H.

Taylor, Hayden

Tcherdyntsev, Victor V.

Tchoukov, Plamen

Teghil, Roberto

Tehranchi, Amirhossein

Teixeira, Jorge
Tejedor, José Antonio Gómez

Telegdi, Judit

Telukutla, Srinivasa Reddy

Teodorescu, Florina

Teodorescu, Valentin Şerban

Terao, Ken

Tereshina-Chitrova, E.A.

TERMENTZIDIS, Konstantinos

Teymourian, Hazhir

Thai Duy, Le

Thakur, Garima

Thakur, Sachin

Thakur, Vijay Kumar

Thang, Tran Quyet

Theres Baby, Tessy

Thijssen, Jos

Thomas, Iorwerth O.

Thomas, T.J.

Thrivikraman, Greeshma

Thygesen, Kristian Sommer

Tibbetts, Katharine Moore

Tiberto, Paola

Tiemann, Michael

Tijing, Leonard

Tikhonravov, Alexander

Tiller, Joerg C.

Timlin, Jerilyn A.

Timperman, Laure

Tiseanu, Carmen

Titvinidze, Irakli

Tkach, Alexander

Tkachenko, Nikolai

Tobaldi, David Maria

Toci, Guido

Tofan, Lavinia

Toffanin, Stefano

Toietta, Gabriele

Tojo, Concha

Tokumoto, Yuki

Tolochko, Oleg

Tomalia, Donald

Tombelli, Sara

Tomer, Vijay K. 


\author{
Tomita, Satoshi \\ Tomita, Shunsuke \\ Tomm, Jens \\ Tomov, Rumen I. \\ Tomovska, Radmila \\ Tomšič, Brigita \\ Toncelli, Claudio \\ Toncheva, Antoniya \\ Tondi, Gianluca \\ Tonelli, Domenica \\ Tonti, Dino \\ Topoglidis, Emmanuel \\ Topuz, Fuat \\ Torgersen, Maria Lyngaas \\ Torigoe, Kanjiro \\ Torii, Shuichi \\ Torimoto, Tsukasa \\ Tornabene, Francesco \\ Torre, Maria Luisa \\ Torreggiani, Armida \\ Torres, Daniel \\ Torres-Lagares, Daniel \\ Torsten, Granzow \\ Tortora, Luca \\ Totaro, Grazia \\ Totaro, Massimo \\ Toudert, Johann \\ Townley, Helen E. \\ Toyao, Takashi \\ Traini, Tonino \\ Trammell, Scott A. \\ Tran, Fabien \\ Tretiakov, Konstantin \\ Tribello, Gareth \\ Trindle, Carl \\ Trinh, Thuat \\ Tripathi, Jitendra Kumar \\ Tripathi, Kumud Malika \\ Trivedi, Rahul \\ Trivellin, Nicola \\ Trocha, Piotr \\ Trubiani, Oriana \\ Trukhanov, Alexei
}

Trukhanov, Sergei

Truong, Nghia P.

Truong, Vi-Khanh

Trzeciak, Anna

Tsai, Chen-Yen

Tsai, Pei-Wei

Tsamis, Christos

Tsay, Chien-Yie

Tseng, Hui-hsin

Tseng, Zong-Liang

Tsitsilianis, Constantinos

Tso, Chi Yan

Tsoi, James Kit-hon

Tsou, Nien-Ti

Tsow, Francis (Tsing)

Tsujimoto, Takashi

TSUTSUI, Makusu

Tsuyumoto, Isao

Tsuzuki, Takuya

Tsvetkov, Nikolai

Tsyganenko, Alexey A.

Tubaro, Cristina

Tudorache, Florin

Tudoroiu, Nicolae

Tulliani, Jean Marc

Tumarkin, Andrey

Tumuluri, Uma

Tung, Shih-Huang

Tung, Tran Thanh

Turco, Antonio

Turco, Rosa

Turkowski, Volodymyr M.

Turner, Neill

Tuszynski, Jack A.

Tuti, Simonetta

Tuttle, Blair

Tyliszczak, Bożena

Tylkowski, Bartosz

Tzetzis, Dimitrios

Tzschucke, Christoph

Uccelletti, Daniela

Uchida, Satoshi

Uchida, Tetsuya 
Uddin, Ashraf

Ueda, Taro

Ujihara, Masaki

Ukrainczyk, Neven

Ul Haq, Rizwan

Ulewicz, Malgorzata

Um, Han-Don

Umek, Polona

Umerska, Anita

Umrath, Stefan

Unnithan, Ranjith Rajasekharan

Unno, Masafumi

Ur, Soon-Chul

Urban, Otmar

Urbanczyk-Lipkowska, Zofia

Urbani, Maxence

Urbassek, Herbert

Urbieta, Ana

Usov, Nikolai

Usui, Hidetomo

Uznanski, Pawel

Vacca, Annalisa

Vaculovičová, Marketa

Vadahanambi, Sridhar

Vadas, Timothy M.

Vahabi, Henri

Vaiano, Vincenzo

Vaidyanathan, Sriram

Vakros, John

Valais, Ioannis

Valentini, Federica

Vallée, Christophe

Valov, Ilia

Van Dyke, Michael W.

van Ommen, J. Ruud

Van Schepdael, Ann

Van Straalen, Nico M.

Vancaeyzeele, Cédric

Vanetsev, Alexander

Vankayala, Raviraj

Varadwaj, Pradeep R.

Varikuti, Sanjay

Varma, Rajender S.
Varsano, Daniele

Vashist, Arti

Vasilache, Dan

Vasilakaki, Marianna

Vasile, Bogdan Stefan

Vasile, Cornelia

Vasilyev, Alexander

Vasquez, Yolanda

Vassilis J, Inglezakis

Vattikuti, Surya Veerendra Prabhakar

Vaz, Carlos

Vaz, Irene Pina

Vaz-Moreira, Ivone

Vecino, Xanel

Vedarajan, Raman

Vedraine, Sylvain

Védrine, Jacques

Vejpravova, Jana

Vekinis, George

Velasco-Vélez, Juan Jesús

Velazquez-Perez, Jesus E

Veleeparambil, Manoj

Velíšek, Josef

Velonia, Kelly

Venditti, Iole

Veremchuk, Igor

Vereshchaka, Alexey A.

Verestiuc, Liliana

Verma, Amit

Verma, Sanny

Vermorel, Romain

Vernardou, Dimitra

Veronesi, Giulia

Veronis, Georgios

Vertruyen, Benedicte

Vescan, Andrei

Vetter, Stefan W.

Veziroglu, Salih

Vicenzo, Antonello

Victor J., Rico

Videira, Romeu A.

Vidic, Jasmina

Vieru, Dumitru 
Vijayaraghavan, Venkatesh

Vikraman, Dhanasekaran

Vilà, Anna

Vilanova, Xavier

Vilardi, Giorgio

Villaverde, Juan José

Viña Olay, Jaime

Vinardell, Maria

Vinci, Giuliana

Vinnik, Denis

Vinogradov, Vladimir

Vinokurov, Vladimir

Virginie, PONSINET

Viskadourakis, Zacharias A.

Viter, Roman

Vitiello, Giuseppe

Vitrik, Oleg Borisovich

Vittori Antisari, Marco

Vittorio, Orazio

Vivas Mejia, Pablo

Viviani, Massimo

Vivo, Paola

Vlachos, Nicholas

Vlachou, Marilena

Vladyslav, Vakarin

Vlaskin, Mikhail

Vohra, Varun

Voicu, Stefan Ioan

Voliani, Valerio

Volk, David

Volker, Körstgens

Volkov, Yuri

Volochaev, V. A.

Voronov, Andriy

Vorontsov, Alexander

Vourdas, Nikolaos

Voutetaki, Maria-Styliani

Voyiatzis, George

Vozzi, Giovanni

Vranes, Milan

Vrcek, Ivana Vinkovic

Vriesekoop, Frank

Wachowski, Sebastian
Wachtveitl, Josef

Wada, Tohru

Waite, Matthew M.

Wakiya, Naoki

Wal, R. L. Vander

Walach, Wojciech

Walde, Peter

Walia, Sumeet

Wallace, David R.

Wallace, Kenneth N.

Wang, Chih-Feng

Wang, Chiu-Yen

Wang, Di-Yan

Wang, Dong Hwan

Wang, Jiangxin

Wang, Shapeng

Wang, Songcan

Wang, Tao

Wang, Xiaolei

Wang, Xiaowei

Wang, Yu

Wang, Yun-Che

Wark, Alastair W.

Wark, Michael

Warwick, Michael E. A.

Wąsik, Tomasz J.

Wasilewski, Tomasz

Watanabe, Fumiya

Watanabe, Masahito

Watanabe, Masatoshi

Watkins, A. Neal

Watras, Adam

Watzky, Murielle

Wautier, Jean-Luc

Wdowin, Magdalena

Webber, Grant

Weber, Matthieu

Węglarz, Władyslaw

Wei, Gang

Weichert, Jamey

Weigand, R.

Weiss, Richard

Weller, Michael G. 
Wen, Amy M.

Whitby, Catherine P

White, Mark

Whitehead, Debra E.

Widenmeyer, Marc

Wie, Myung-Bok

Wieczorek, Kinga

Wieczorek, Władysław G.

Wiemann, Martin

Wieszczycka, Karolina

Wiglusz, Rafał J.

Wilhelm, Rene

Wilkins, Richard T

William Coleman, Anthony

Williams, Gareth

Wilson, Andrew J.

Wilts, Bodo

Winkler, K

Wiśniewski, Marek

Wittemann, Alexander

Witzel, Bernd

Włodarczyk, Patryk

Wojcieszak, Robert

Wojcik, Janusz

Wojnarowicz, Jacek

Wong, Ka-Leung

Wong, Patrick

Wong, Roong Jien

Won-Yeop, Rho

Woo, Yun Sung

Woodward, Robert $\mathrm{T}$.

Worrall, Stephen

Worsley, Marcus A.

Wróbel, Jan

Wu, Chia-Wen

$\mathrm{Wu}$, Donghai

Wu, Mao-Sung

$\mathrm{Wu}, \mathrm{Yu}$-Tse

Wujcik, Evan K.

Wuttke, Stefan

Wycisk, Ryszard

Wyman, Ian

Wysokowski, Marcin
Xia, Lan

Xie, Haibo

Xifré-Pérez, Elisabet

$\mathrm{Xu}$, Tingting

$\mathrm{Xu}$, Xiaoxue

Yadav, Hemraj M

Yadav, Raghvendra Singh

Yadav, Sushma

Yadavalli, Nataraja Sekhar

Yadgarov, Lena

Yagoubi, Saïd

Yahiaoui, Riad

Yakimov, E.B.

Yakimova, Rositsa

Yalcinkaya, Fatma

Yamada, Toshishige

Yamaguchi, Aritomo

Yamaguchi, Makoto

Yamaguchi, Seiji

Yamamoto, Go

Yamamoto, Yusuke

Yamato, Takehiko

Yamauchi, Yusuke

Yáñez-Sedeño, Paloma

Yáñez-Vilar, Susana

Yang, C.-F.

Yang, En-Che Yang

Yang, Hsiharng

Yang, Jen-Chang

Yang, Lily

Yano, Mitsuaki

Yao, Qiaofeng

Yasui, Kyuichi

Ye, Long

Yee, Ki-Ju

Yehezkeli, Omer

Yentekakis, Ioannis

Yeo, Boon Siang

Yeom, Junghoon

Yeom, Junseok

Yerushalmi-Rosen, Rachel

Yeum, Jeong

Yi, Gi-Ra 


\begin{tabular}{|c|c|}
\hline Yildirim, Adem & Zanotti, Jean-Marc \\
\hline Yilmaz, Mehmet & Zappa, Dario \\
\hline Yokota, Shingo & Zaraska, Leszek \\
\hline Yoo, Dong Jin & Zarejousheghani, Mashaalah \\
\hline Yoo, Dongwon & Zarifi, Mohammad H. \\
\hline Yoon, Dae Sung & Zarkadoula, Eva \\
\hline Yoon, Hyeonseok & Zarrelli, Mauro \\
\hline Yoon, Minyoung & Zavaleta, Cristina \\
\hline Yoon, Sorah & Zawala, Jan \\
\hline Yoshimatsu, Kohei & Zazpe, Raul \\
\hline Yoshimoto, Makoto & Zekonyte, Jurgita \\
\hline Yoshino, Toshihiko & Żelechowska, Kamila \\
\hline You, Jungmok & Zelzer, Mischa \\
\hline You, Shujie & Zemek, Josef \\
\hline Younis, Adnan & Zeng, Huang \\
\hline Yperman, Jan & Zeng, Shuwen \\
\hline Yu, Hakki & Zergioti, Ionna \\
\hline Yu, Jae Woong & Zhang, Qiangzhe \\
\hline Yu, Jiashing & Zhang, R. Y. \\
\hline $\mathrm{Yu}, \mathrm{Lu}$ & Zhang, Run \\
\hline Yu, Taekyung & Zhang, Xiang \\
\hline Yu, Xiao-Ying & Zhang, Xin \\
\hline Yuan, Kuo & Zhang, Xuefeng \\
\hline Yuba, Eiji & Zhang, Yue \\
\hline Yudasaka, Masako & Zhao, Donggao \\
\hline Yuguchi, Yoshiaki & Zhao, Lianfeng \\
\hline Yuldashev, Sh. & Zhao, Yuda \\
\hline Yum, Jun Ho & Zheng, Xinting \\
\hline Yun, Kyusik & Zhi, Chunyi \\
\hline Yun, Seok Joon & Zhitova, Elena \\
\hline Yun, Young Soo & Zhou, Yiqun \\
\hline Yurukcu, Mesut & Zhu, Can \\
\hline Yusenko, Kirill V. & Zhu, Jiadeng \\
\hline Zabihi, Omid & Zhu, Jie \\
\hline Zaccheroni, Nelsi & Zhuravlev, Victor \\
\hline Zacharatos, Filimon & $\mathrm{Zi}$, Yunlong \\
\hline Zaitsu, Shin-ichi & Ziegler-Borowska, Marta \\
\hline Zakharenko, A.M. & Zielinska, Beata \\
\hline Zamani, Akram & Zielińska-Jurek, Anna \\
\hline Zamaratskaia, Galia & Zieliński, Andrzej \\
\hline Zambon, Daniel & Ziemkowska, Wanda \\
\hline Zandvliet, Harold & Zignani, Sabrina Campagna \\
\hline Zannotti, Marco & Zille, Andrea \\
\hline
\end{tabular}


Ziora, Zyta

Zitka, Ondrej

Zografopoulos, Dimitrios C.

Zolfagharian, Ali

Zubia, David

Zubrik, Anton
Zukowski, Witold

Zuo, Lijian

Zuorro, Antonio

Zur, Lidia

Żyła, Gaweł

(C) 2020 by the author. Licensee MDPI, Basel, Switzerland. This article is an open access article distributed under the terms and conditions of the Creative Commons Attribution (CC BY) license (http://creativecommons.org/licenses/by/4.0/). 